\title{
Monte Carlo Studies of Magnetic Nanoparticles
}

\author{
K. Trohidou and M. Vasilakaki \\ Computational Materials Science Group, Institute of Materials Science, \\ National Center of Scientific Research 'DEMOKRITOS', \\ Patriarhou Grigoriou \& Neapoleos St. 153-10 Agia Paraskevi, Athens,
}

Greece

\section{Introduction}

Magnetic nanoparticles are complex mesoscopic systems which have unique physical properties that clearly differ from those of atoms and bulk materials. They find numerous technological applications ranging from ultra-high-density recording media (Bader, 2006) to biomedicine (Pankhurst et al., 2003). The necessity to reduce the size of the nanoparticles for these applications have raised a key issue in their study which is their thermal stability.

The Monte Carlo (MC) simulation technique with the implementation of the Metropolis Algorithm (Metropolis et al., 1953) has been proved a very powerful tool for the systematic study of the magnetic behaviour of nanoparticles and nanoparticle assemblies. The two major advantages of this technique are a) the possibility for atomic scale treatment of the nanoparticles, so the details of their microstructure can be studied and b) the implementation of finite temperature through the Metropolis algorithm.

Although, the obtained dynamics in the Monte Carlo simulations is intrinsic and the time evolution of the system does not come from any deterministic equation for the magnetisation, the results of the Monte Carlo simulations reproduce qualitatively the trend of the experimental data (Binder 1987). Actually this good qualitative agreement between the simulation results and the experimental data enable us to have a better insight into the nanoscaled phenomena, though some of them stem from non-equilibrium processes (Landau \& Binder, 2000).

A microscopic treatment of the magnetisation of ferromagnetic nanoparticles, using Monte Carlo techniques, was first developed by Binder and co-workers (Binder et al., 1970; Wildpaner 1974). An important demonstration of the work was the reduction of the magnetisation near the surface of the particle. Clearly this was to be expected because a surface spin has a smaller number of neighbours than it would have in bulk and, hence, experiences a reduced mean field. For very small particles (less than say $5 \mathrm{~nm}$ ) the proportion of surface spins is such that they will make a major contribution to the magnetisation. As a result, the magnetisation will decrease with temperature over a range where the bulk magnetisation is roughly constant and deviations from Curie-law behaviour in the susceptibility are to be expected. In the period following the Monte Carlo work cited above, interest has been developed in finite-size scaling, and it is in this context that subsequent advances (Landau, 1976) in the nanoparticle magnetism have occurred. In addition, over the last decade there is a continuous effort to reduce the nanoparticles size and at the same time to overcome the thermal instability at room temperature (Skumryev et 
al., 2003). This led to the study of composite nanoparticle magnetic structures with core/shell morphology.

In what follows we will review our MC simulation results for atomic scale modelling on spherical ferromagnetic (FM), antiferromagnetic (AFM), ferrimagnetic (FI) and composite nanoparticles with core/shell morphology. The magnetisation dependence on external parameters (temperature, applied field) and the intrinsic particle properties (size, size of shell and core, size and type of anisotropy, magnetic structure) are studied. Finite size effects and the role of the surface will be discussed for the FM, AFM and FI nanoparticles. In the case of the composite nanoparticles, which consist of a spherical ferromagnetic core surrounded by an antiferromagnetic (or ferrimagnetic) shell, we examine the effect of the interface between the ferromagnetic core and the shell of the particles, on their magnetic properties.

Finally our MC simulations results on the influence of the interparticle interactions on the macroscopic magnetic behaviour of assemblies of nanoparticles will be reviewed. The characteristics of the hysteresis loop and the temperature dependent magnetisation (Field Cooled (FC)/ Zero-Field Cooled (ZFC)) are studied numerically in magnetic nanoparticle assemblies using Monte Carlo simulations and the standard Metropolis algorithm. The computational technique for the calculation of the long ranged interparticle interactions will be discussed and results will be given for granular assemblies and ordered arrays of magnetic nanoparticles.

A discussion on potential applications and a comparison with experimental findings will be given in all cases.

\section{Metropolis Monte Carlo simulation for the magnetic nanoparticles}

The MC simulation technique is a standard method to study models of equilibrium or non equilibrium thermodynamic systems with many degrees of freedom by stochastic computer simulation. The starting point of the simulations is the appropriate choice of a model Hamiltonian and then the use of random numbers to simulate statistical fluctuations in order to generate the correct thermodynamical probability distribution according to a canonical ensemble (Binder 1986, 1987). In this way one may obtain microscopic information about complex systems which cannot be studied analytically or which might not be accessible in a real system. Contrary to Landau-Lifshitz or Langevin equations, Monte Carlo scheme provides the straightforward implementation of the temperature.

To simulate the magnetic nanoparticles and the nanoparticle assemblies and to derive thermodynamic averages, the elementary physical quantity that we use is the spin. In the case of the single nanoparticles we consider a classical spin at each atomic site and we simulate using the MC technique the stochastic movement of the system in the phase space. In the case of assemblies, we consider an effective spin to represent the magnetic state of each nanoparticle (Stoner \& Wohlfarth, 1948).

The MC simulation consists of many elementary steps. In every elementary step a spin $\hat{S}_{i_{\text {old }}}$ is randomly chosen from a system of $N$ spins and an attempted new orientation $\hat{S}_{\text {inew }}$ of the spin is generated with a small random deviation $\widehat{\delta S}$. The attempted direction is chosen in a spherical segment around the present orientation $\hat{S}_{i_{\text {old }}}$. Then the energy difference $\Delta \mathrm{E}$ between the attempted and the present orientation is calculated. In the Metropolis Monte Carlo algorithm, if $\Delta \mathrm{E}<=0$ the new orientation is accepted, if $\Delta \mathrm{E}>0$ the attempted new orientation is accepted provided that a random number $\mathrm{u}$, generated uniformly in the 
interval $(0,1)$, is less than the probability $\exp \left(-\Delta \mathrm{E} / \mathrm{k}_{\mathrm{B}} \mathrm{T}\right)$, otherwise the system remains to its present state (Binder, 1987). A complete MC step (MCS) consists of $N$ elementary steps so that in any MC step on average every spin is considered once. With this algorithm, states are generated with a certain probability (Importance Sampling) and rejecting the first MCS that correspond to the thermalization process the desired average of a variable, namely the sum of the products of each value times the corresponded probability, simply become arithmetic average over the entire sample of states which is kept.

One common problem that appears during the MC simulation is that if we draw the attempted direction of every spin independently of the previous one, the system will always be superparamagnetic and no hysteresis will result, since it will be possible to explore the whole phase space independently of the temperature and due to the large fluctuations in every MCS it will escape very quickly from any metastable state responsible for hysteresis. By fixing to a certain limit the deviation $\widehat{\delta S}$, it is possible to modify the range of acceptance and model the real system more accurately (Garscia-Otero et al., 1999; Dimitrov \& Wysin, 1996; Binder 1987) than choosing $\hat{S}_{\text {inew }}$ completely randomly and independently from $\hat{S}_{i_{\text {old }}}$. The MC acceptance rate can be set to some desired value (40-60\%) (setting effectively the rate of motion in phase space). The use of such a kind of local dynamics permits to detect confinement in metastable states which are responsible for the hysteresis and to achieve true relaxation in different temperatures. Therefore we choose to perform the Metropolis MC in such a way that it samples the phase space "locally" with accepted ratio $50 \%$.

Over the years several modified MC methods have been proposed to treat the problem of overcoming the local minima during the $\mathrm{MC}$ numerical procedure depending on the details of the system (e.g. Chantrell et al., 2001; Hinzke \& Nowak, 1999; H. F. Du \& A. Du , 2006). However the MC Metropolis algorithm works fast and efficiently in all cases.

In order to avoid trapping of the system at local minima, we start the numerical procedure from an unmagnetised sample at a high temperature above the critical temperature of the sample and we reduce the temperature gradually at a constant rate. At the temperatures above $T_{c}$ we use more $M C$ steps than at the lower temperature to let the system relax surpassing probable metastable states. Special care has been taken of the time and ensemble averaging of the magnetisation of the system by properly choosing the number of MC steps and a rather big number of different samples namely independent random number sequences corresponding to different realizations of thermal fluctuations.

The thermodynamic quantities that we calculate with the use of the MC Metropolis algorithm in the magnetic nanoparticles and their assemblies are the coercive field, the remanent magnetisation and the $\mathrm{ZFC} / \mathrm{FC}$ magnetisation curves.

The coercive field $\left(\mathrm{H}_{\mathrm{c}}\right)$ are defined as the magnetic field required to reverse the magnetisation of the particle. In order to obtain the coercive field we calculate the complete hysteresis loop. A Field Cooling procedure is performed initially. Once the desired temperature is reached, we calculate the loop starting from the positive saturation and slowly decreasing the applied field in very small constant steps. In each value of the field, several MCS are executed, then the magnetisation is calculated and the field is changing again. We continue to reduce the field so the system goes to its negative saturation state. Then we increase again gradually the field until the system reaches its positive saturation. In this way a complete hysteresis loop is performed. The remanent magnetisation $\left(\mathrm{M}_{\mathrm{r}}\right)$ is taken at the zero field point of the descending magnetisation versus field curve.

The ZFC/FC magnetisation curves is obtained by the following steps: a) initially we start with the sample at very high temperature (above its critical temperature) and we 
gradually reduce the temperature down to a very low value (close to zero), to obtain its ground state, b) at this very low temperature we apply a magnetic field and we start raising the temperature up to the maximum value that we had started, in this way we obtain the ZFC curve, c) finally in the presence of the magnetic field we reduce the temperature gradually down to the minimum value and in this way we obtain the FC magnetisation curve.

We have kept constant step rate of the magnetic field in the calculation of the hysteresis loops and of the temperature in the calculation of the $\mathrm{ZFC} / \mathrm{FC}$ magnetisation curves (see e.g. Bahiana et al., 2004).

\section{Model Hamiltonians for the magnetic nanoparticles and nanoparticle assemblies.}

\subsection{Isolated magnetic nanoparticles}

The Monte Carlo simulations are performed using the Metropolis algorithm as described in section 2. For the energy calculation of the single particle systems we use the following models:

a) In the case of FM, AFM and FI nanoparticles, we consider spherical nanoparticles with radius $R$, expressed in lattice spacings, on a simple cubic lattice. The outer layer of one lattice spacing is considered to be the surface of the nanoparticle in all cases. The spins in the particle interact with nearest neighbours Heisenberg exchange interaction, and at each crystal site they experience a uniaxial anisotropy. In the presence of an external magnetic field, the total energy of the system is:

$$
E=-J \sum_{<i, j>} \vec{S}_{i} \cdot \vec{S}_{j}-K_{c} \sum_{i \in \text { core }}\left(\vec{S}_{i} \cdot \hat{e}_{i}\right)^{2}-K_{s} \sum_{i \in \text { srf }}\left(\vec{S}_{i} \cdot \hat{e}_{i}\right)^{2}-\vec{H} \cdot \sum_{i} \vec{S}_{i}
$$

Here $S_{i}$ is the atomic spin at site $i$ and $\hat{e}_{i}$ is the unit vector in the direction of the easy axis at the site $i$. The first term gives the exchange interaction between the spins, the second is the anisotropy energy of the core, the third gives the anisotropy energy of the surface and the last term is the Zeeman energy. The core anisotropy $\left(K_{c}\right)$ is assumed uniaxial along the zaxis. The surface anisotropy $\left(\mathrm{K}_{\mathrm{s}}\right)$ is considered either radial or random. The exchange coupling constant $\mathrm{J}$ for the FM nanoparticles is taken equal to one, for the AFM ones -1 and for the FI ones -1.5. The hysteresis loops are calculated after a Field Cooling procedure as described in Section 2.

b) For the composite spherical nanoparticles with FM core and an AFM shell or FI shell in a simple cubic lattice, we take into account explicitly the exchange interaction between the spins in the core, the interface, the shell and the surface considering also in this case nearest neighbours Heisenberg exchange interactions (Eftaxias \& Trohidou, 2005; Eftaxias et al., 2007; Vasilakaki \& Trohidou, 2009). The energy of the system is given as:

$$
\begin{aligned}
& E=-J_{F M} \sum_{<i, j \in F M>} \vec{S}_{i} \cdot \vec{S}_{j}-J_{S H} \sum_{<i, j \in S H>} \vec{S}_{i} \cdot \vec{S}_{j}-J_{I F} \sum_{<i, j \in I F>} \vec{S}_{i} \cdot \vec{S}_{j} \\
& -\sum_{i \in F M} K_{i F M}\left(\vec{S}_{i} \cdot \hat{e}_{i}\right)^{2}-\sum_{i \in S H} K_{i S H}\left(\vec{S}_{i} \cdot \hat{e}_{i}\right)^{2}-\vec{H} \cdot \sum_{i} \vec{S}_{i}
\end{aligned}
$$

The first, second and third terms give the core, shell and interface exchange interaction respectively. The exchange coupling constant $\mathrm{J}_{\mathrm{FM}}$ for the core spins is taken equal to one. We 
set the exchange coupling constant of the $\mathrm{J}_{\mathrm{SH}}=-\mathrm{J}_{\mathrm{FM}} / 2$. The exchange coupling constant of the interface $\mathrm{J}_{\mathrm{IF}}$ is equal to $\mathrm{J}_{\mathrm{SH}}$ in size and the interaction is taken ferromagnetic. The fourth term gives the anisotropy energy of the FM core. If the site i lies in the outer layer of the FM core, the anisotrophy is defined as $\mathrm{K}_{\mathrm{iFM}}=\mathrm{K}_{\mathrm{IF}}$ and $\mathrm{K}_{\mathrm{iFM}}=\mathrm{K}_{\mathrm{C}}$ elsewhere. The anisotropy always is considered uniaxial along the z-axis in the core and along the interface. The fifth term gives the anisotropy energy of the AFM shell and it is considered either along the z-axis or random. If $i$ lies in the outer layer of the shell then the anisotropy is defined as $\mathrm{K}_{\mathrm{iSH}}=\mathrm{K}_{\mathrm{S}}$ and $\mathrm{K}_{\mathrm{iSH}}=\mathrm{K}_{\mathrm{SH}}$ elsewhere. The surface anisotropy is taken as random. So $\mathrm{K}_{\mathrm{C}}, \mathrm{K}_{\mathrm{IF}}, \mathrm{K}_{\mathrm{SH}}, \mathrm{K}_{\mathrm{S}}$, denote the core, the interface, the shell and the surface anisotropy respectively. The last term gives the Zeeman energy.

We simulate a Field Cooling procedure starting at a temperature which is between the Curie temperature $\mathrm{T}_{\mathrm{c}}$ of the ferromagnetic core and the critical temperature of the antiferromagnetic or ferrimagnetic shell; consequently we cool the nanoparticle at a constant rate in the presence of a magnetic field $\mathrm{H}_{\text {cool }}$ along the $\mathrm{z}$-axis. The resulting hysteresis loops have a horizontal and vertical asymmetry. The value of the loop shift along the field axis is expressed by the exchange bias field $\mathrm{H}_{\mathrm{ex}}=-\left(\mathrm{H}_{\mathrm{right}}+\mathrm{H}_{\text {left }}\right) / 2$, and the coercive field is defined as $\mathrm{H}_{\mathrm{c}}=\left(\mathrm{H}_{\text {right }}-\mathrm{H}_{\text {left }}\right) / 2, \mathrm{H}_{\text {right }}$ and $\mathrm{H}_{\text {left }}$ being the points where the loop intersects the field axis. The vertical shift (DM) that expresses the asymmetry along the magnetisation axis, is given as $D M=\left(M_{u p}-M_{\text {down }}\right) / 2, M_{u p}$ and $M_{\text {down }}$ being the points where the loop intersects the M-axis. $M_{r}$ is normalized to the magnetisation at saturation $\left(M_{s}\right)$.

\subsection{Assemblies of magnetic nanoparticles}

We considered two types of nanoparticle assemblies: three-dimensional (3D) randomly placed magnetic nanoparticle assemblies and quasi two-dimensional (2D) ordered arrays of magnetic nanoparticles.

In the first type we consider spherical nanoparticles with diameter D located randomly inside a cubic box with edge length equal to $\mathrm{L}$. The particle assembly is assumed monodisperse. To avoid the overlap problem, the space inside the box is discretised by a simple (or face centered) cubic lattice with lattice constant equal to the particle diameter. The magnetic state of each particle is described, according to the Stoner-Wohlfarth model (Stoner \& Wohlfarth, 1948), by a classical spin vector $\left(\overrightarrow{\mathrm{S}_{\mathrm{i}}}\right)$ with an anisotropy axis in a random direction $\left(\hat{\mathrm{e}}_{\mathrm{i}}\right)$. The particles interact via long range dipolar forces and via exchange forces, when they are sufficiently close. The total energy of the assembly is given by equation 3 :

$$
E=g \sum_{i, j} \frac{\left(\hat{S}_{i} \cdot \hat{S}_{j}\right)-3\left(\hat{S}_{i} \cdot \hat{R}_{i j}\right)\left(\hat{S}_{j} \cdot \hat{R}_{i j}\right)}{R_{i j}^{3}}-J \sum_{<i, j>} \hat{S}_{i} \cdot \hat{S}_{j}-k \sum_{i}\left(\hat{S}_{i} \cdot \hat{e}_{i}\right)^{2}-h \sum_{i}\left(\hat{S}_{i} \cdot \hat{H}\right)
$$

where $\hat{S}_{i}$ is the direction of the spin of the nanoparticle $i, \hat{e}_{i}$ is the easy axis direction, $R_{i j}$ is the centre-to-centre distance between particles $i$ and $j$, measured in units of the particle diameter and hats indicate unit vectors. The first term gives the dipolar energy where $g$ is the dipolar strength defined as $g=\mu^{2} / D^{3}$. The second term gives the exchange energy with exchange strength $\mathrm{J}$. The third term gives the anisotropy energy with the anisotropy constant defined as $k=K_{1} V_{o}$ and the last term gives the Zeeman energy with $h=\mu H$ where $\mu=M_{s} V_{o}$ is the nanoparticle magnetic moment and $V_{o}$ the nanoparticle volume. The exchange coupling exists only between particles in contact (nearest neighbours). 
The particles occupy at random the sites within the cube with an occupation probability $p$. The MC cell is repeated periodically and the Ewald summation technique is implemented to calculate the long range part of the dipolar energy (Kretschmer \& Binder, 1979). Satisfactory convergence with the Ewald technique is obtained using repetitions of the central Monte Carlo cell along each of the three Cartesian axes (Kechrakos \& Trohidou, 1998). As a test of convergence of the Ewald series we calculated the value of the local field at a site of a fully aligned ferromagnetic state of a crystalline $(p=1)$ assembly. The theoretical value $\mathrm{H}_{0}=(4 \Pi / 3)$ $\mathrm{M}_{\mathrm{s}}$ was reproduced with accuracy $10^{-4}$.

For the model of the quasi 2D nanoparticle ordered arrays we consider identical spherical particles with diameter $\mathrm{D}$ forming a two-dimensional triangular lattice in the xy-plane and lattice constant $d \geq D$. We construct a nanoparticle-assembled film with finite thickness (1-4 monolayers (ML)) by placing particles in the upper layer above alternate interstices in the lower one (Puntes et al., 2001). Structural defects are considered only in the uppermost ML. The nanoparticles are single-domain, they posses uniaxial anisotropy in a random direction and they interact via dipolar forces. The total energy of the system is given again by equation (3); in this case the exchange interaction term is not taken into account, since in the ordered arrays the nanoparticles are not in contact (Kechrakos \& Trohidou, 2002). We used periodic boundaries in the xy-plane and free boundaries in the z-axis. The dipolar interactions were treated without truncation using the Ewald summation method for a quasi-two-dimensional system (Grzybowski et al., 2000).

In all cases of nanoparticles and nanoparticle systems, the fields $\mathrm{H}_{\mathrm{cool}}$ and $\mathrm{H}_{\mathrm{c}}$ are given in units of $\mathrm{J} / \mathrm{g} \mu_{\mathrm{B}}$, the temperature $\mathrm{T}$ in units $\mathrm{J} / \mathrm{k}_{\mathrm{B}}$, and the anisotropy coupling constants $\mathrm{K}$ in $\mathrm{J}$. In our simulation we have used from 18.000 up to 40.000 Monte Carlo steps per spin (Binder 1987), depending on the system size. The results are averaged over 10-30 samples with different spin configurations for the single particles and the assemblies and in the case of the random assemblies different spatial configurations for the nanoparticles have also been considered.

\section{MC simulation results for isolated FM, AFM and FI nanoparticles.}

Surface effects, resulting basically from the symmetry breaking of the lattice, and finite-size effects have a strong influence on the magnetic properties of single-domain nanoparticles. As the particle size decreases in FM, AFM and FI nanoparticles, new or modified magnetic properties come to light opening new horizons for research, production of novel nanostructures and technological applications. A common feature to the study of single domain nanoparticles has been the large deviations from the uniform reversal magnetisation of the Stoner-Wohlfarth model and the existence of a progressive switching of the spins that caused the need for a different numerical treatment including micromagnetic details describing the surface effects.

Our Monte Carlo simulations have proved to be efficient to examine the influence of the surface spins and to incorporate the surface effects to the magnetic behaviour of ferromagnetic, antiferromagnetic and ferrimagnetic nanoparticles with uniaxial, random and radial surface anisotropy.

\subsection{Ferromagnetic nanoparticles}

FM nanoparticles show enhanced magnetic moments and enhanced effective magnetic anisotropy values as the size decreases. This has been associated with the influence of the 
surface atoms that become more significant with decreasing size due to increasing surface to volume ratio (Chen et al., 1998; Respaud et al., 1998; Bødker et al., 1994; Jamet et al., 2001). Also a decrease of the net surface magnetisation has been attributed to the effect of increasing surface disorder or surface spin canting or even to the existence of a dead magnetic layer (Curiale et al., 2009).

We have calculated the magnetisation of FM particles by the MC simulation technique using equation (1) for the energy minimisation. The results in the absence of any surface anisotropy are shown in figure 1(a) for two spherical nanoparticles. In this figure the temperature dependence of the magnetisation is shown for particles with uniform uniaxial anisotropy $\mathrm{K}_{\mathrm{c}}$ with the easy axis along the $\mathrm{z}$-direction, $\mathrm{K}=\mathrm{K}_{\mathrm{c}}=\mathrm{K}_{\mathrm{s}}=0.1 \mathrm{~J}$ (full symbols). For these ferromagnetic particles, the decrease in the magnetisation with decreasing $R$ is well known and it is ascribed to the increasing role played by the surface as $\mathrm{R}$ becomes smaller (Trohidou et al., 1998 a, 1998 b; Gangopadhyay et al., 1992; Dimitrov \& Wysin, 1994). The effect of introducing radial surface anisotropy is next considered, for these two particles (open symbols). The surface anisotropy $\mathrm{K}_{\mathrm{s}}$ is one order of magnitude higher than the core $\mathrm{K}_{\mathrm{c}}=0.1 \mathrm{~J}, \mathrm{~K}_{\mathrm{s}}=10 \mathrm{~K}_{\mathrm{c}}$ with the easy axis normal to the surface at each site. We observe in this case a reduction of the magnetisation due to the surface disorder introduced by the radial anisotropy and a more rapid fall of the magnetisation with temperature. The radial direction of the easy axis orientation of the strong surface anisotropy when averaged over the whole surface of the spherical particle tends to eliminate the contribution to the magnetisation from the surface layer. This behaviour is in agreement with experimental findings on $\left(\mathrm{Fe}_{0.26} \mathrm{Ni}_{0.74}\right)_{50} \mathrm{~B}_{50}$ nanoparticles (De Biasi et al., 2002), on metallic Fe nanoparticles (Bodker et al., 1994) and Fe nanoparticles (Chen et al., 1998).

In figure $1(b)$ the coercive field versus temperature for these two nanoparticles is displayed. Full symbols represent results for uniform $\mathrm{z}$-axis anisotropy $\mathrm{K}=\mathrm{K}_{\mathrm{c}}=\mathrm{K}_{\mathrm{s}}=0.1 \mathrm{~J}$ and open symbols for $\mathrm{z}$-axis core anisotropy $\mathrm{K}_{\mathrm{c}}=0.1 \mathrm{~J}$ and radial surface anisotropy of size $\mathrm{K}_{\mathrm{s}}=10 \mathrm{~K}_{\mathrm{c}}$. The observed behaviour for the particles with uniform anisotropy is the predicted one from the phenomenological model of Kneller and Luborsky (Kneller \& Luborsky, 1963). The bigger particle has the higher coercivity and this behaviour is valid for all temperatures.
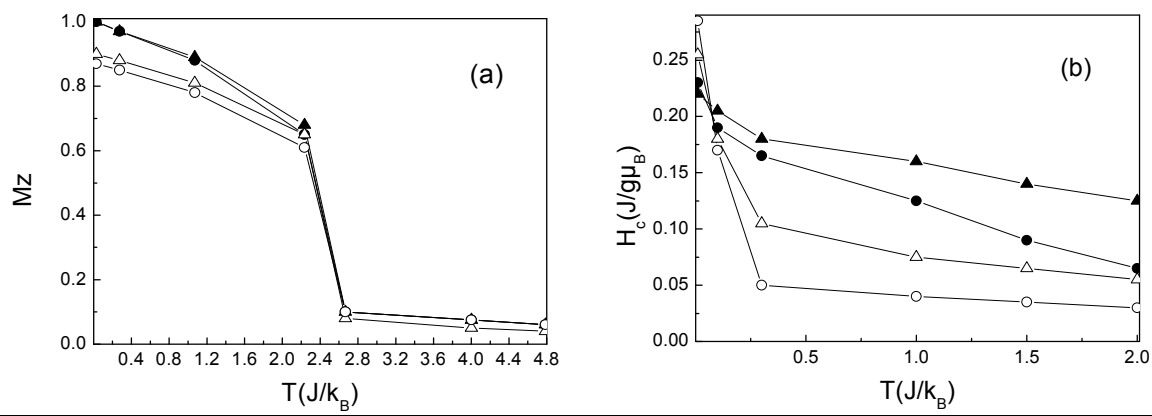

Fig. 1. Magnetisation versus temperature (a) and $\mathrm{H}_{\mathrm{c}}$ versus temperature (b) for ferromagnetic particles with sizes: $R=8.0$ (circles); $R=12.0$ (triangles) with uniform uniaxial anisotropy $\mathrm{K}=0.1 \mathrm{~J}$ (full symbols) and with radial surface anisotropy $\mathrm{K}_{\mathrm{s}}=10 \mathrm{~K}_{\mathrm{c}}$ (open symbols). 
The behaviour of the particles with the radial surface anisotropy is quite different. Increase of the low temperature coercivity for the particles with radius $\mathrm{R}=8$ is observed and an inversion with the size. As the temperature increases this behaviour is reversed, also we observe a steeper drop with temperature than in the uniform anisotropy case in agreement with Garanin \& Kachkachi, 2003. This is due to the fact that the thermal fluctuations mask the surface contribution.

\subsection{Antiferromagnetic nanoparticles}

AFM nanoparticles have attracted major interest since the pioneering study of Néel (Néel, 1953). The imbalance of the spin population on the antiparallel sublattices (uncompensated spins denoted by $\mathrm{N}_{\mathrm{u}}$ ) gives a finite moment to the nanoparticles. Néel (Néel, 1953) first pointed out that the uncompensated spins of the AFM nanoparticles are lying on the surface (Trohidou, 2005). Experimental findings on AFM nanoparticle systems showed deviations of the magnetisation curves from the Langevin function above the blocking temperature, low blocking temperatures, shifted loops and high coercivities in the low temperature regime (Kodama R H 1999; Mørup \& Hansen 2005). These findings indicate the interplay between size and surface effects and they can be described by a core / shell model where the nanoparticle consists of an antiferromagnetically ordered core and a disordered surface shell. This shell represents the frustrated magnetic state at the surface (Winkler et al., 2005; Bhowmik et al., 2004).

We have simulated four AFM spherical nanoparticles with very similar sizes but different numbers of uncompensated spins ( $R=7.5$ lattice spacings $N=1791$ and $N_{u}=79, R=7.75$ with $\mathrm{N}=1935$ and $\mathrm{N}_{\mathrm{u}}=17, \mathrm{R}=8.1$ with $\mathrm{N}=2205$ and $\mathrm{N}_{\mathrm{u}}=83$ and $\mathrm{R}=8.5$ with $\mathrm{N}=2553$ and $\mathrm{N}_{\mathrm{u}}=25$ ), $\mathrm{N}$ is the total number of spins in the nanoparticle. We introduce uniaxial anisotropy for the core spins and a strong random anisotropy at the surface to simulate the experimentally observed spin-glass like phase. The parameters for the anisotropy strength are $\mathrm{K}_{\mathrm{c}}=0.1 \mathrm{~J}$ in the core and $\mathrm{K}_{\mathrm{s}}=1.5 \mathrm{~J}$ at the surface (Vasilakaki \& Trohidou, 2008).

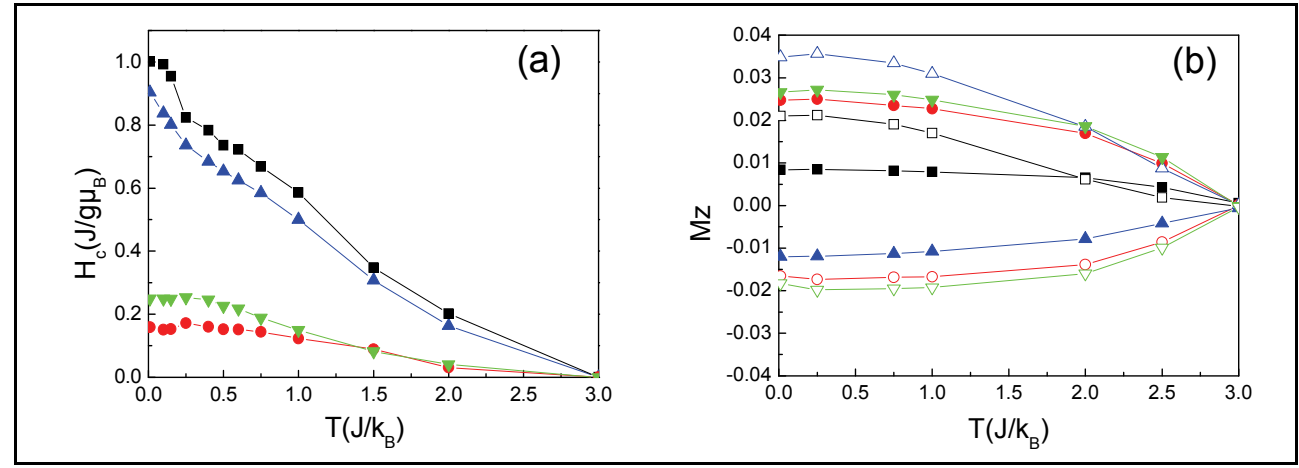

Fig. 2. Temperature dependence $(\mathrm{a})$ of the coercive field $\left(\mathrm{H}_{\mathrm{c}}\right)$ and $(\mathrm{b})$ of the $\mathrm{z}$ component of the magnetisation $(\mathrm{Mz})$ in the core (full symbols) and at the surface (open symbols) for AFM nanoparticles of radii $\mathrm{R}=7.5$ (squares), $\mathrm{R}=7.75$ (circles), $\mathrm{R}=8.1$ (up triangles) and $\mathrm{R}=8.5$ (down triangles) lattice spacings with random surface anisotropy.

By implementing the Monte Carlo simulation technique and the Metropolis algorithm for the energy minimization (given in eq. 1), we calculated the coercive field and the magnetisation versus temperature (figure 2). We observe that the $\mathrm{H}_{\mathrm{c}}(\mathrm{T})$ curves are very close 
for the nanoparticles with similar size of $\mathrm{N}_{\mathrm{u}}$. Also it appears that at intermediate temperatures they follow the $\mathrm{N}_{\mathrm{u}} / \mathrm{N}$ scaling law as discussed in (Néel, 1953; Trohidou, 2005). In figure 2(b) we give the temperature dependence of the core (full symbols) and the surface (open symbols) contributions to the $\mathrm{z}$ component of the magnetisation for the four nanoparticles. It can be seen clearly that the coercive field follows the surface behaviour.

For comparison in figure 3 we present results for the same nanoparticles for radial surface anisotropy and the same anisotropy strengths as in the random surface anisotropy case. The most striking feature in this figure is the appearance of a peak of the $\mathrm{H}_{\mathrm{c}}$ at the same temperature roughly for all the nanoparticles. From figure 3(b) we can see that this is the temperature where the surface spins have a peak in their magnetisation so the surface spins drag the core ones and this accounts for the peak in the coercive field. Then as the temperature increases the surface spins move due to thermal fluctuations causing the gradual decrease of magnetisation. This behaviour is in agreement with that of layered systems with competing interactions (Leighton et al., 2002). It is apparent from figure 2(a) that also in this case of anisotropy the $\mathrm{H}_{\mathrm{c}}$ scales with the $\mathrm{N}_{\mathrm{u}} / \mathrm{N}$ ratio at intermediate temperatures.
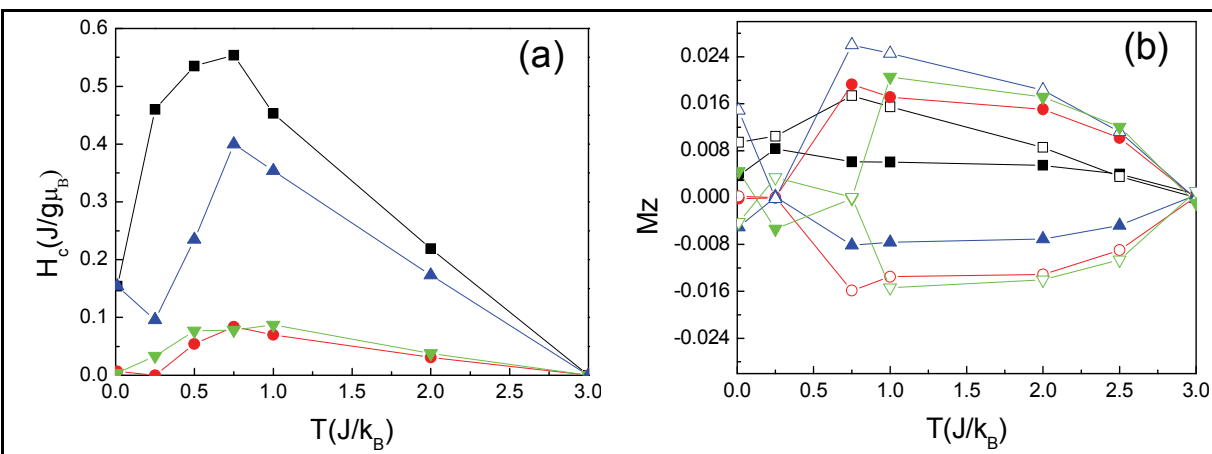

Fig. 3. Temperature dependence (a) of the coercive field $\left(\mathrm{H}_{\mathrm{c}}\right)$ and $(\mathrm{b})$ of the $\mathrm{z}$ component of magnetisation (Mz) in the core (full symbols) and at the surface (open symbols), for AFM nanoparticles of radii $\mathrm{R}=7.5$ (squares), $\mathrm{R}=7.75$ (circles), $\mathrm{R}=8.1$ (up triangles) and $\mathrm{R}=8.5$ (down triangles) lattice spacings with radial surface anisotropy.

The negative surface contribution in figures 2(b) and 3(b) from the surface magnetisation in the nanoparticle with radii $\mathrm{R}=7.75$ and 8.5 lattice spacings and very small number of uncompensated spins $\left(\mathrm{N}_{\mathrm{u}}=17,25\right)$ is due to the non-uniform distribution of the small number of surface spins as discussed in (Trohidou et al., 1998). Also we observe in all nanoparticles a small increase of the core magnetisation in both cases of anisotropy at finite temperatures due to the fact that as temperature increases we have contribution from sublattice spins in the magnetisation as it has been discussed in (Mørup et al., 2007; Brown et al., 2005).

We also examined how the different surface anisotropy strengths modify the coercive field behaviour as a function of temperature (Vasilakaki \& Trohidou, 2008). Our simulations demonstrated that the $\mathrm{H}_{\mathrm{c}}(\mathrm{T})$ behaviour depends on the relative $\mathrm{K}_{\mathrm{s}} / \mathrm{K}_{\mathrm{c}}$ ratio. The decreasing surface anisotropy allows the core spins to drag continuously the surface ones as they become disordered with increasing temperature. Our results are in agreement with the experimental findings of (Winkler et al., 2008) on noninteracting $\mathrm{NiO}$ nanoparticles. 


\subsection{Ferrimagnetic nanoparticles}

In the case of ferrimagnetic nanoparticles also the surface role becomes important with the decrease of the size (Iglesias \& Labarta, 2004; Leite et al., 2005; Martinez et al., 1998) and even larger than the antiferromagnetic surface because the net magnetic component of the ferrimagnetic surface is larger. The idea also of a noncollinear spin arrangement at the surface responsible for the moment reduction was proposed very early (Coey, 1971).

In the case of ferrimagnetic nanoparticles we discuss the effect of the uncompensated spins on their coercive behaviour. We use the same parameters for the particle sizes, the core and the surface anisotropy as in the AFM nanoparticles for comparison.

In figure 4(a) we give the results for the coercive field versus temperature and in figure 4(b) we have plotted the temperature dependence of core and the surface contributions to the $\mathrm{z}$ component of the magnetisation for the four FI nanoparticles for random surface anisotropy and in figures $5(\mathrm{a})$ and $5(\mathrm{~b})$ for radial surface contribution. At temperatures close to $\mathrm{T}=0$ $\mathrm{J} / \mathrm{k}_{\mathrm{B}}$, it is the core that contributes to the coercive behaviour of the biggest particles and we have an almost identical contribution of the surface for the two smaller ones. As a result all four nanoparticles have the same coercive field. As the temperature increases we have some contribution from the surface of the bigger nanoparticle and this gives a slower decrease of the coercive field with temperature for the bigger ones. This can be clearly seen from the magnetisation behaviour of the core and the surface in figure 4(b). The larger nanoparticles at very low temperature have negligible surface contribution to the magnetisation in comparison to the smaller ones. As the temperature increases the competition between the spin canting and the thermal fluctuations causes an increase in the surface contribution to the magnetisation for these nanoparticles. The details of this surface contribution depend on the distribution of the uncompensated spins on the surface of the particles. The magnetisation behaviour observed in figure $4(\mathrm{~b})$ is in agreement with the magnetisation behaviour of $\mathrm{Fe}_{2} \mathrm{O}_{3}$ nanoparticles in (Kachkachi et al., 2000).

The dependence of the magnetisation and the coercive field with temperature does not change when we replace the random anisotropy with radial at the surface confirming that here the magnetic behaviour is less sensitive to the surface contribution as it can be seen from figures 5 .
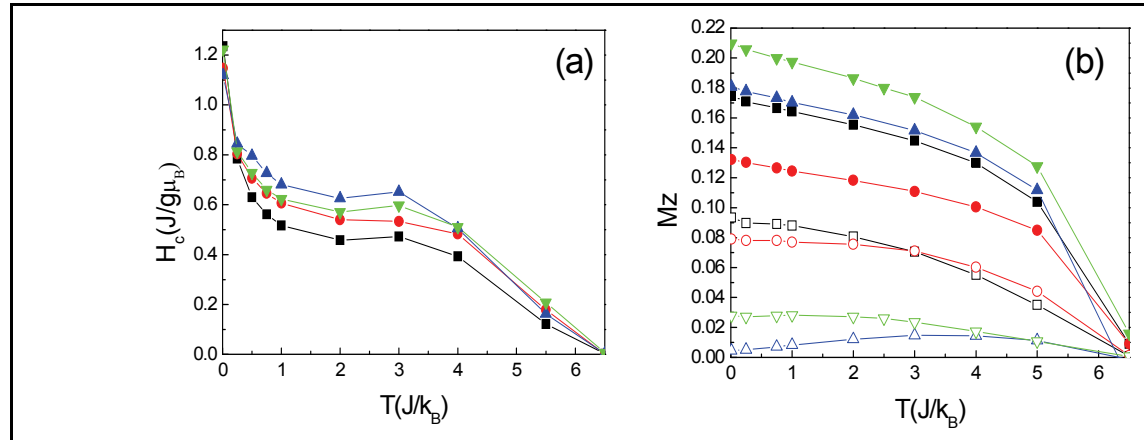

Fig. 4. Temperature dependence (a) of the coercive field $\left(\mathrm{H}_{\mathrm{c}}\right)$ and $(\mathrm{b})$ of the $\mathrm{z}$ component of the magnetisation $(\mathrm{Mz})$ in the core (full symbols) and at the surface (open symbols) for FI nanoparticles of radii $\mathrm{R}=7.5$ (squares), $\mathrm{R}=7.75$ (circles), $\mathrm{R}=8.1$ (up triangles) and $\mathrm{R}=8.5$ (down triangles) lattice spacings with random surface anisotropy. 

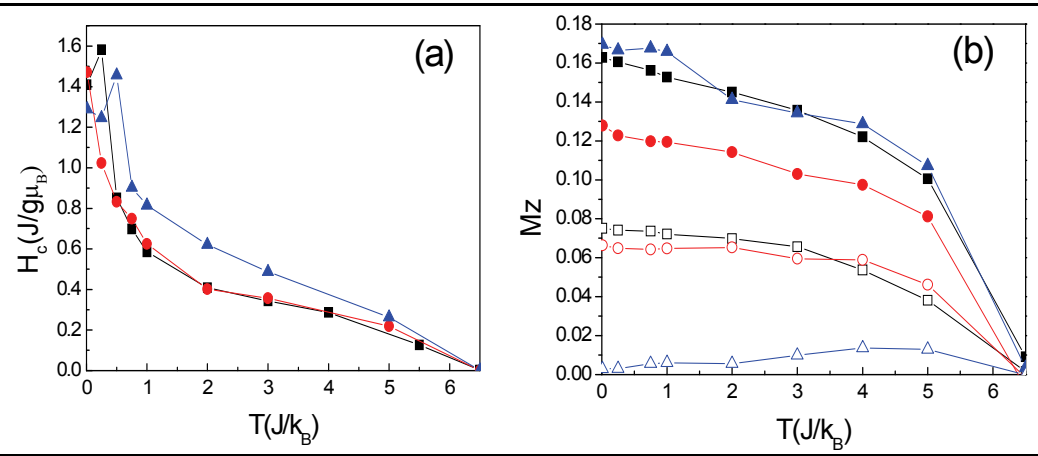

Fig. 5. Temperature dependence (a) of the coercive field $\left(\mathrm{H}_{\mathrm{c}}\right)$ and $(\mathrm{b})$ of the $\mathrm{z}$ component of the magnetisation (Mz) in the core (full symbols) and at the surface (open symbols) for FI nanoparticles of radii $\mathrm{R}=7.5$ (squares), $\mathrm{R}=7.75$ (circles) and $\mathrm{R}=8.1$ (up triangles) lattice spacings with radial surface anisotropy.

\section{MC results for composite magnetic nanoparticles with FM core / AFM or FI shell morphology.}

Fine particles with core/shell morphology have attracted great research interest due to rich and often unusual magnetic properties (Nogues et al., 2005). They exhibit enhancement of the coercive field and thermal stability at very small sizes, properties desirable for permanent magnets and the high density magnetic recording materials.

These properties are observed in all magnetic nanostructured materials with two different spin structures in contact. They exhibit asymmetry, on the magnetic field axis, of their hysteresis loops which is caused by a unidirectional anisotropy, the exchange anisotropy, induced by the exchange coupling at the interface between the different spin structures, when they are cooled down in a static magnetic field $\left(\mathrm{H}_{\text {cool }}\right)$. The effect was first observed in field cooled $\mathrm{Co} / \mathrm{CoO}$ composite nanoparticles with ferromagnetic core and antiferromagnetic (AFM) shell morphology (Meiklejohn \& Bean, 1957) and it is known as the exchange bias effect. Although in the decades that followed the exchange bias research focused mainly on thin film systems, the production of magnetic nanoparticles with core/shell morphology and their study have renewed interest in the exchange bias phenomena (Nogues et al., 2005).

In addition to the exchange bias shift a vertical shift (DM) of the hysteresis loops has also been observed in FM/AFM core/shell nanoparticles attributed to the uncompensated spins of the shell (Passamani, 2006).

We have investigated the exchange bias mechanism and the factors that influence the exchange bias behaviour in FM core/AFM shell nanoparticles. In our studies (Zianni \& Trohidou, 1998; Eftaxias et al., 2007) on FM core/AFM shell nanoparticles, we used the MC simulation technique employing the Metropolis algorithm to study the factors that influence their exchange bias behaviour. We found (Eftaxias \& Trohidou, 2005; Eftaxias et al., 2007) that the exchange bias field at very low temperature is approximately constant after the second AFM layer, a result which is in agreement with the experimental findings of (Morel et al., 2004) where a very fast stabilization of the exchange bias field with oxygen dose in $\mathrm{Co} / \mathrm{CoO}$ nanoparticles is observed and in $\mathrm{Co} / \mathrm{CoO}$ nanoparticles embedded in an $\mathrm{Al}_{2} \mathrm{O}_{3}$ 
matrix (Nogues et al., 2006). As the temperature increases, more AFM layers are needed to increase and stabilize the exchange bias field, because the thermal fluctuations mask the interface contribution, and therefore a thicker shell is required to stabilize the interface contribution; and after a certain number of AFM layers, roughly when the shell size becomes initially equal in size to the core and then further increases, the exchange bias field is decreasing because of the enhancement of the AFM contribution that masks the interface role.

We next studied isolated composite nanoparticles with a FM core and FI disordered shell morphology, in order to investigate the underlying mechanism for the exchange bias effects in these systems. The anisotropy constant for the core is $\mathrm{K}_{\mathrm{C}}=0.05 \mathrm{~J}_{\mathrm{FM}}$, for the ferromagnetic interface $\mathrm{K}_{\mathrm{IF} / \mathrm{FM}}=0.5 \mathrm{~J}_{\mathrm{FM}}$ one order of magnitude larger than $\mathrm{K}_{\mathrm{c}}$, for the ferrimagnetic interface, the shell and the surface: $\mathrm{K}_{\mathrm{IF} / \mathrm{FI}}=1.5 \mathrm{~J}_{\mathrm{FM}}, \mathrm{K}_{\mathrm{SH}}=1.5 \mathrm{~J}_{\mathrm{FM}}$ and $\mathrm{K}_{\mathrm{s}}=1.5 \mathrm{~J}_{\mathrm{FM}}$ respectively. We introduce the strong random anisotropy in the shell and at surface in order to simulate a spin-glass like phase.

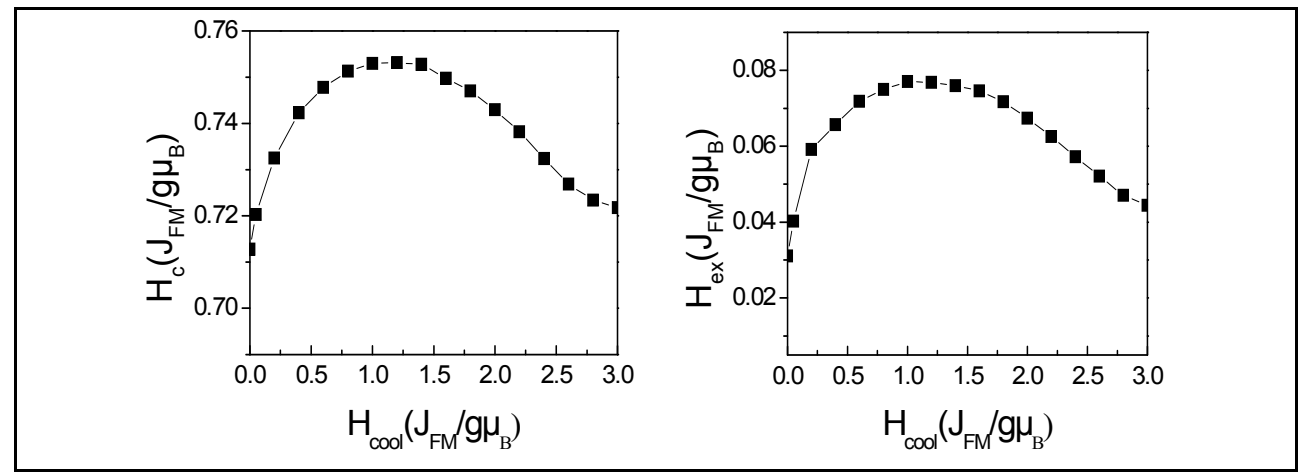

Fig. 6. Cooling field dependence of the coercive field $\left(\mathrm{H}_{c}\right)$, and the exchange bias field $\left(\mathrm{H}_{\mathrm{ex}}\right)$ for a nanoparticle with core size 5 lattice spacings and shell thickness 7 lattice spacings.

The cooling field dependence of the $\mathrm{H}_{\mathrm{ex}}, \mathrm{H}_{\mathrm{c}}$ and $\mathrm{M}_{\mathrm{r}}$ is given in figure 6 as a function of the applied cooling field $\mathrm{H}_{\text {cool }}$ for a nanoparticle with FM core 5 lattice spacings and FI shell 7 lattice spacings. Initially as the $\mathrm{H}_{\text {cool }}$ increases, it causes an increase in both $\mathrm{H}_{\mathrm{ex}}$ and $\mathrm{H}_{\mathrm{c}}$. The gradual increase of $\mathrm{H}_{\text {cool }}$ tends to align a certain amount of FI spins at the interface along the field direction. After some $\mathrm{H}_{\text {cool }}$ value further increase in the cooling field, results in a decrease of these two quantities. For these higher cooling field values the Zeeman coupling between the field and the FI spins dominates the magnetic interactions inside the system. So the FI spins follow the applied field and as a result the exchange bias field and the coercive field decrease. Our MC results reproduced very well the behaviour of the cooling field dependence of $\mathrm{H}_{\mathrm{c}}, \mathrm{H}_{\mathrm{ex}}$ and $\mathrm{M}_{\mathrm{r}}$ of $\mathrm{Fe} / \mathrm{FeO}$ nanoparticles systems in (Baker et al, 2004; Del Bianco et al, 2004).

The influence of the shell thickness in the behaviour of the hysteresis loop, starting the field cooling procedure with a field $\mathrm{H}_{\text {cool }}=0.4 \mathrm{~J} / \mathrm{g} \mu_{\mathrm{B}}$ in nanoparticles with FM core/FI shell is discussed. We consider four particles with total radii $\mathrm{R}=9.0, \mathrm{R}=12.0, \mathrm{R}=14.0$ and $\mathrm{R}=20.0$ lattice spacings. They all have the same core size of 5 lattice spacings and FI shell thickness 4, 7, 9 and 15 lattice spacings respectively. The surface thickness is one lattice spacing, in all cases. The results for the hysteresis loops for these four particles are shown in figure 7(a) at a 
very low temperature $\mathrm{T}=0.01 \mathrm{~J}_{\mathrm{FM}} / \mathrm{k}_{\mathrm{B}}$. As we can see the hysteresis loops are shifted and the nanoparticles with the bigger shell thickness have the bigger shift and the bigger coercive field. So both $\mathrm{H}_{\mathrm{c}}$ and the $\mathrm{H}_{\mathrm{ex}}$ increase with the shell thickness while $\mathrm{M}_{\mathrm{r}}$ decreases. This is in agreement with the experimental findings of Baker and his collaborators on $\mathrm{Fe} / \mathrm{FeO}$ nanoparticles (Baker et al., 2004). We also observe a small vertical shift in the hysteresis loops of the two nanoparticles with the smaller shell thickness. The asymmetry in the magnetisation axis disappears for the two bigger nanoparticles. The hysteresis loop for the nanoparticle with the lower shell thickness has a shoulder, characteristic of a two-phase system. This shoulder disappears as the shell thickness increases, because the shell dominates in the hysteresis behavior of the sample. In figure 7(a) we observe that the hysteresis loops of the nanoparticles with the bigger shell thickness are less saturated, due to the enhancement of the spin-glass like behaviour (Binder \& Young, 1986).

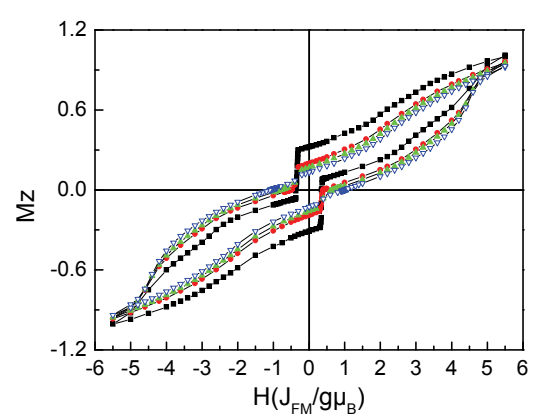

(a)

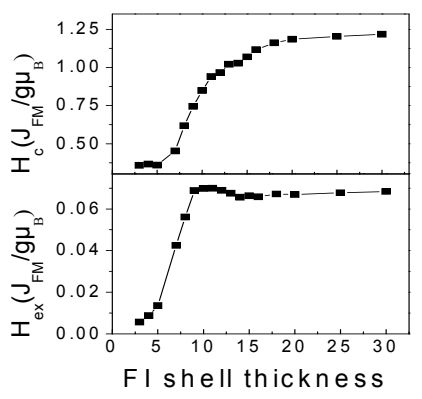

(b)

Fig. 7. (a) Hysteresis loops of core/shell nanoparticles with core radius $R_{c}=5$ lattice spacings and shell thickness 4(squares), 7(circles), 9(up triangles), 15(down triangles) lattice spacings respectively (b) Shell thickness dependence of the coercive field $\left(\mathrm{H}_{c}\right)$, the exchange bias field $\left(\mathrm{H}_{\mathrm{ex}}\right)$.

In figure $7(b) H_{c}$ and $\mathrm{H}_{\mathrm{ex}}$ have been plotted as a function of the shell thickness. We observe that: a) the coercive field increases continuously with the shell thickness, $b$ ) the $\mathrm{H}_{\text {ex }}$ increases slowly with the increase of the shell thickness and then it remains constant.

The increase of $\mathrm{H}_{\mathrm{c}}$ with the increase of the disordered layer is expected due to the fact that the thicker shell has bigger number of disordered spins. Our MC results agree with the experimental findings for $\mathrm{Fe} / \mathrm{Fe}$ oxide core/ shell nanoparticles by (Baker et al., 2004). In experimental studies of FM/AFM (Nogues \& Schuller, 1999) or FM/FI bilayers (Lin et al., 1994) and $\mathrm{Co} / \mathrm{CoO}$ (Peng et al., 2000) nanoparticles, they found that $\mathrm{H}_{\mathrm{ex}}$ increases and then saturates for large AFM thickness. In the case of the ferrimagnetic shell nanoparticles it needs more layers even at low temperature in comparison with the AFM shell case(Eftaxias et al., 2007) for the appearance of the exchange bias effects.

The core size dependence of the exchange bias field and the coercive field is next considered. In figure 8 we present results for the $\mathrm{H}_{\mathrm{c}}$ and $\mathrm{H}_{\mathrm{ex}}$ versus temperature for three nanoparticles with the same shell thickness of 7 lattice spacings and three different core sizes of 3, 5 and 10 lattice spacings. As we can see, at very low temperature smaller core radius have the bigger coercive and exchange bias field, in agreement with the experimental findings of (Del Bianco et al., 2003). This is due to the fact that the biggest contribution from 
the interface is obtained in the nanoparticle with the smallest core radius. The coercive field is decreasing faster with temperature in the case of the smaller core nanoparticles than in the bigger ones. There is a crossing temperature above which the behaviour is changed and the nanoparticles with the bigger core radius have higher coercivity. This is the temperature at which the shell becomes totally disordered. Above this temperature the coercivity follows the temperature dependence of the core. The smaller in size core becomes faster superparamagnetic. This is in agreement with experimental findings by (S. Gangopadhyay et al., 1992) on Fe nanoparticles surrounded by a disordered iron oxide shell. In the case of the two smaller nanoparticles the $\mathrm{H}_{\mathrm{c}}$ decays exponentially as in the case of the varying shell thickness (see Fig. 7) due to the dominance of the disordered shell. For the biggest nanoparticle $\mathrm{H}_{\mathrm{c}}$ has a monotonic temperature dependence due to the dominant ferromagnetic character. The exchange bias field vanishes very quickly with the increase of the temperature.

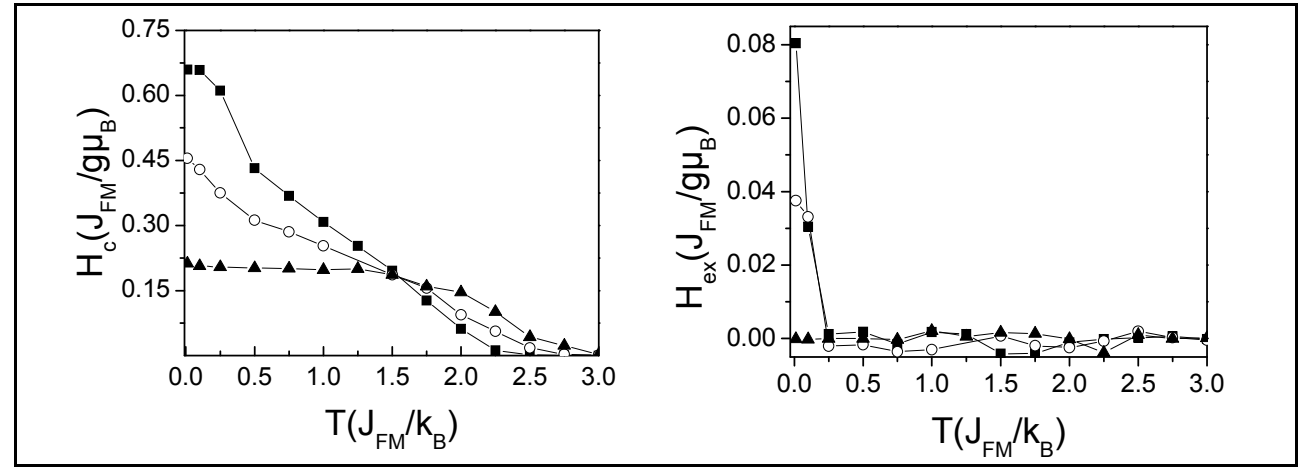

Fig. 8. $\mathrm{H}_{\mathrm{c}}$ and $\mathrm{H}_{\mathrm{ex}}$ as a function of temperature for nanoparticles with shell thickness 7 lattice spacings and core 3(squares), 5(circles) and 10(triangles) lattice spacings respectively.

For the case of composite nanoparticles with spin glass-like shell the aging and training effect on the $\mathrm{H}_{\mathrm{c}}$ and $\mathrm{H}_{\mathrm{ex}}$ have been also studied. These effects are present in the FM core /FI spin glass-like shell systems, since one of the characteristics of spin glass systems is their multiple energy configuration of the ground state (Binder \& Young, 1986). So the frozen spins, which are originally aligned in the cooling field direction, may change their directions and fall into other metastable configurations during the hysteresis measurements. This characteristic of spin-glass-like phase essentially influences the exchange bias behaviour of the system and results in a decrease of $\mathrm{H}_{\mathrm{c}}$ and $\mathrm{H}_{\mathrm{ex}}$ with the field cycling. The behaviour of the training effect for the composite FM core/FI spin glass-like shell nanoparticles will depend on its microstructure characteristics. In figure 9 we have plotted the $H_{e x}$ and $H_{c}$ as a function of the loop cycling for three nanoparticles with core radius 5 lattice spacings and shell thickness 7, 9 and 15 lattice spacings (Vasilakaki \& Trohidou, 2009). After a field cooling process, the hysteresis loop was calculated six consecutive times at temperature $\mathrm{T}=0.01 \mathrm{~J}_{\mathrm{FM}} / \mathrm{k}_{\mathrm{B}}$. We observe that $\mathrm{H}_{\mathrm{ex}}$ in the case of the nanoparticle with shell thickness 7 lattice spacings after the first loop has a big reduction, while $\mathrm{H}_{\mathrm{ex}}$ for the other two nanoparticles has a small reduction with the loop cycling and very similar. These two nanoparticles have the same size of the exchange bias field as it can be seen from figure 6 .

$\mathrm{H}_{\mathrm{c}}$ has a bigger reduction with the loop cycling for the smaller shell nanoparticle than in the other two. This behaviour indicates that the interface has the major contribution in the 
training effect, for the chosen nanoparticles. As the shell thickness decreases we expect contribution from the shell and the core to the training effect. So a $\sim 50 \%$ reduction of $\mathrm{H}_{\mathrm{ex}}$ during cycling has been observed in FM/FI nanoparticles (Peng et al., 2002; Trohidou et al., 2007) with small shell thickness. Whereas a $\sim 12 \%$ reduction in the case of $\mathrm{Co} / \mathrm{CoO}$ nanoparticles (Peng et al, 2000). In any case we expect that the behaviour of the training effect depends not only on the magnetic microstructure but on other factors, in agreement with the experimental observations in FM/AFM bilayer systems (Nogués et al., 2005).
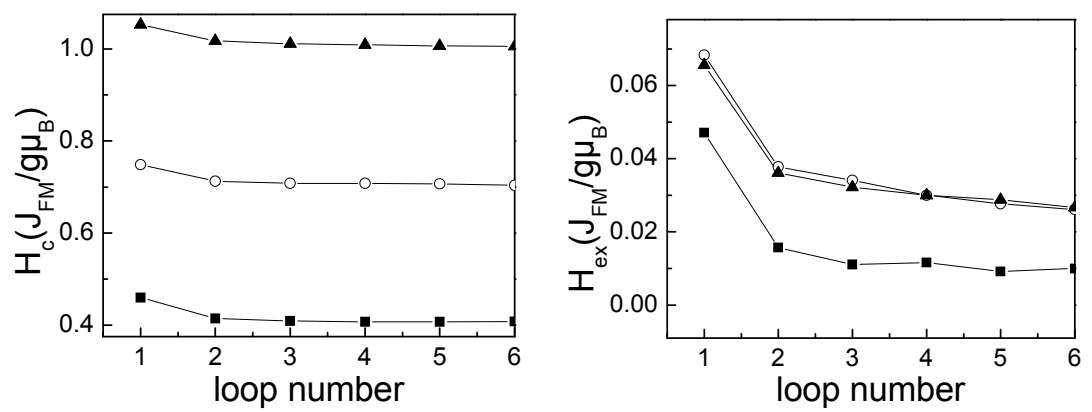

Fig. 9. Training effect of $(\mathrm{a}) \mathrm{H}_{\mathrm{c}}$ and $(\mathrm{b}) \mathrm{H}_{\mathrm{ex}}$ for core/ shell nanoparticles with core size 5 lattice spacings and shell thickness 7(squares), 9(circles) and 15(triangles) respectively.

Nanogranular systems of which one of two phases, that are in contact, is spin-glass like phase are characterized by aging effects below their critical temperature $\left(\mathrm{T}_{\text {glass }}\right)$, (Chamberlin et al., 1984) due to the slow response of the magnetisation with the time evolution. We have studied the aging effect, namely the slowing down of the spin dynamics with increasing the waiting time $\left(t_{\mathrm{w}}\right)$ spent in the frozen state before any field variation for single nanoparticles with a FM core and a spin-glass like FI shell.

In figure 10 we present our MC simulation results for the time dependence of the thermoremanent magnetisation (TRM) for two different waiting times for a nanoparticle of radius 9 lattice spacings (Fiorani et al., 2006). The numerical procedure is the following: the system is cooled from a high temperature with zero field down to an iniatial temperature $\mathrm{T}_{\mathrm{i}}=0.75\left(\mathrm{~J} / \mathrm{k}_{\mathrm{B}}\right)$, then a field $\mathrm{H}_{\text {cool }}=0.4\left(\mathrm{~J} / \mathrm{g} \mu_{\mathrm{B}}\right)$ is applied along the $\mathrm{z}$-axis for different times $t_{w}$ and we continue field cooling at a constant rate down to a low temperature $\mathrm{T}_{\mathrm{f}}=0.15\left(\mathrm{~J}_{\mathrm{FM}} / \mathrm{k}_{\mathrm{B}}\right)$. At this temperature the $\mathrm{H}_{\mathrm{cool}}$ is removed and we calculate the TRM as a function of time expressed in MCS.

We observe that by increasing the waiting time, TRM decays slower with time because the system during the longer waiting time goes to a lower energy configuration and then by reducing the temperature the system remains trapped to this local minimum, as it is more difficult to overcome the energy barriers separating different states. Therefore the relaxation time of the TRM is enlarged.

The slowing down of the spin dynamics with increasing $t_{w}$ has a noticeable effect on the exchange bias properties of single nanoparticles with FM core and a spin-glass like FI shell (Fiorani et al., 2006). Our MC studies demonstrated that the $H_{e x}$ and $M_{r}$ increase with $t_{w}$. This fact is important for technological applications, because by varying the waiting time we can control the exchange bias behaviour of the system. 


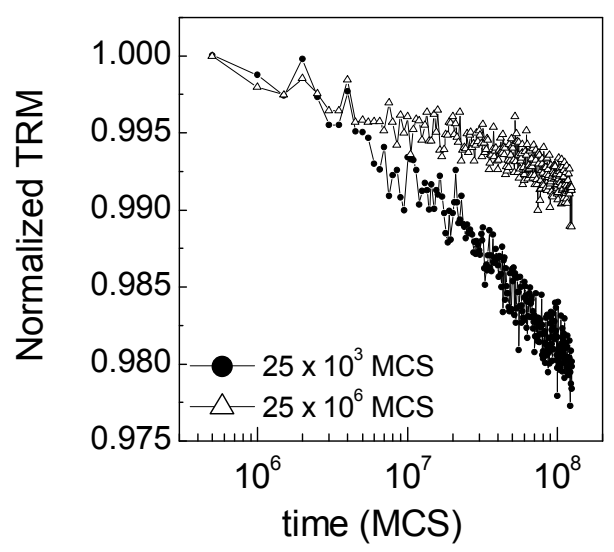

Fig. 10. TRM vs time for two different $t_{w}$ values, as obtained by the MC simulations. TRM is normalized to the value at the beginning of the simulation.

\section{MC simulation results of Interparticle interactions effects on magnetic nanoparticle assemblies}

For decades assemblies of interacting magnetic nanoparticles have been a fascination and a challenge for materials scientists. Magnetic nanoparticles are commonly formed in assemblies, with either random or ordered structure. In the first group belong systems such as ferrofluids and granular solids, while in the second group belong the patterned media (or magnetic dots) and the self-assembled arrays of nanoparticles. In the assemblies of magnetic nanoparticles the crucial role of interparticle interactions in determining their response to an externally applied field as well as the temperature dependence of the magnetic properties has been recognized long ago (Dormann et al., 1997). In this article we review our results from MC simulations of the field and temperature dependence of the magnetisation of nanoparticle assemblies.

\subsection{Random assemblies of magnetic nanoparticles}

In a three dimensional (3D) random assembly of magnetic nanoparticles, especially at high densities, interparticle interactions have an important and sometimes dominant role in the formation of the magnetic behavior. Magnetostatic interactions between the particles are always present owing to the magnetic moment each particle carries. Due to their long range character they cannot be neglected except at the extreme dilute limit. Furthermore, exchange interaction between the particles appears when there is physical contact between them. The exchange interaction is expected to play an important role in samples with concentration close and above the percolation threshold. Indeed as the nanoparticle concentration increases, the interparticle interactions modify the distribution of the effective energy barrier, resulting in more complex phenomena, such as superspin glass (SSG) behaviour in low-enough temperatures for intermediate concentration systems (Sahoo et al., 2003) and superferromagnetic (SFM) order for very dense systems (Bedanta et al., 2007).

The characteristics of the hysteresis loop (remanence and coercivity) and the blocking temperature have been shown to vary with nanoparticle concentration in granular metals 
and frozen ferrofluids (Dormann et al., 1997). The experimental trend was successfully reproduced by a model that includes interparticle dipolar interactions (Kechrakos \& Trohidou, 1998; Chantrell et al., 2001).

We have investigated the role of interparticle exchange in modifying the concentration dependence of the hysteresis characteristics and the blocking temperature of a nanoparticle assembly (Kechrakos \& Trohidou, 2003). The whole range of exchange constants strengths is studied, thus modelling the transition from well separated to coalesced particles.

The concentration dependence of the coercivity is shown in figure 11. In the dilute limit the coercivity at zero temperature is theoretically predicted to approach the value of $\mathrm{H}_{\mathrm{c}}=0.96 \mathrm{~K}_{1} / \mathrm{M}_{\mathrm{s}}$. The data in figure 11 are slightly below this value due to the finite temperature $\left(\mathrm{T}=0.01 \mathrm{~J} / \mathrm{k}_{\mathrm{B}}\right)$ at which the simulation is performed. Beyond the dilute limit, the effect of either type of interactions on the coercivity is quite similar, as the overall decrease of the coercivity values indicate. In the case of dipolar interaction only (full circles) the reduction of coercivity is due to the demagnetizing character of the dipolar forces that is also responsible for the reduction of the remanence. On the other hand, exchange interactions favor the formation of ferromagnetic clusters of particles with low anisotropy, as explained earlier, and therefore the magnetisation reversal is facilitated and the coercivity is reduced relative to the non-interacting case. When both types of interactions are present, we observe that their effect on the coercivity is not additive in all cases. In particular for weak exchange coupling (full circles and triangles) the interactions act cooperatively and the coercivity is further reduced relative to the case of exclusively dipolar or exchange forces. However, in the case of particle coalescence (open stars) the introduction of dipolar forces in the sample of coalesced particles (full stars) shifts the coercivity upwards. We attribute this behavior to the strong random dipolar fields generated in the sample containing large, almost isotropic, coherent clusters of particles. In this case, dipolar forces introduce an extra, albeit weak, anisotropy in the system that enhances the coercive field values.

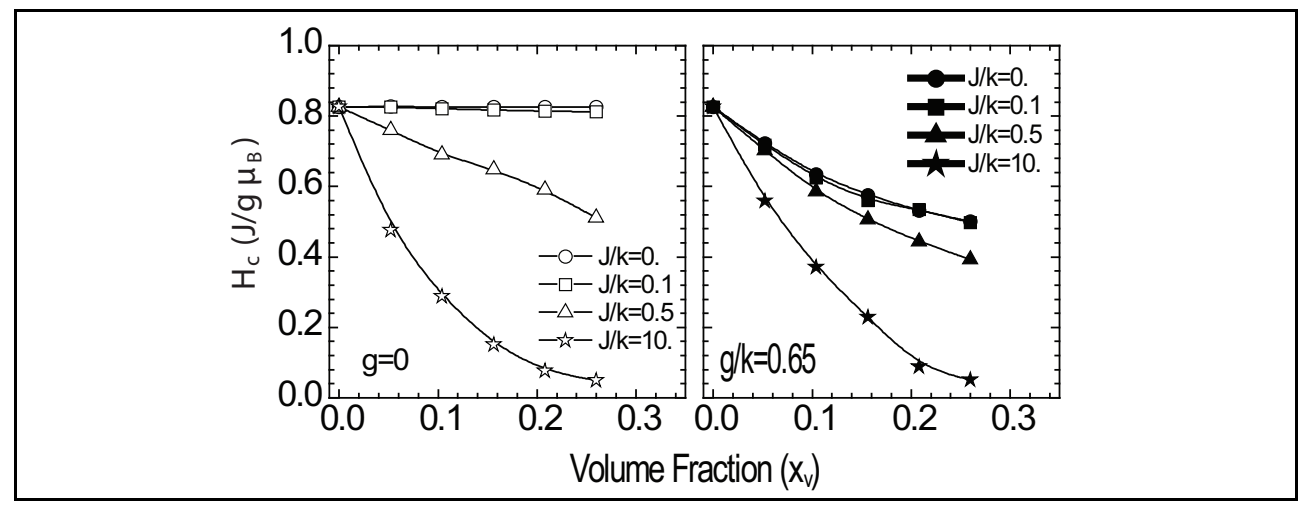

Fig. 11. Concentration dependence of coercivity at low temperature $\left(T=0.01 \mathrm{~J} / \mathrm{k}_{\mathrm{B}}\right),(\mathrm{a})$ exchange coupling only $(\mathrm{g}=0)$, $(\mathrm{b})$ exchange and dipolar coupling $(\mathrm{g} / \mathrm{k}=0.65)$.

We discuss next the temperature dependence of the magnetisation. The Zero-Field-Cooled / Field-Cooled (ZFC/FC) curves are calculated for metal volume fraction $\mathrm{xv}=0.15$. Representative results are shown in figure 12. The peak of the ZFC curve provides the blocking temperature $\left(T_{b}\right)$ of the system (Dormann et al., 1997). Interparticle interactions produce an upshift of $\mathrm{T}_{\mathrm{b}}$ and they modify the high temperature (superparamagnetic) 
behavior of the magnetisation. In particular, Curie's law $(\chi \sim 1 / T)$ that is valid in the case of non-interacting superparamagnetic particles (open circles), is no longer obeyed by the interacting system. The deviations from Curie's law are stronger when both types of interactions are present (full circles). In this case an almost linear decay with temperature is observed. Comparing the effect of the two types of interactions in the high temperature regime, we notice that dipolar interactions produce stronger deviations from Curie's law than exchange interactions and this is attributed to their long-range character. These results are in agreement with experimental findings on Fe nanoparticles in an Ag matrix (Binns et al., 2002).

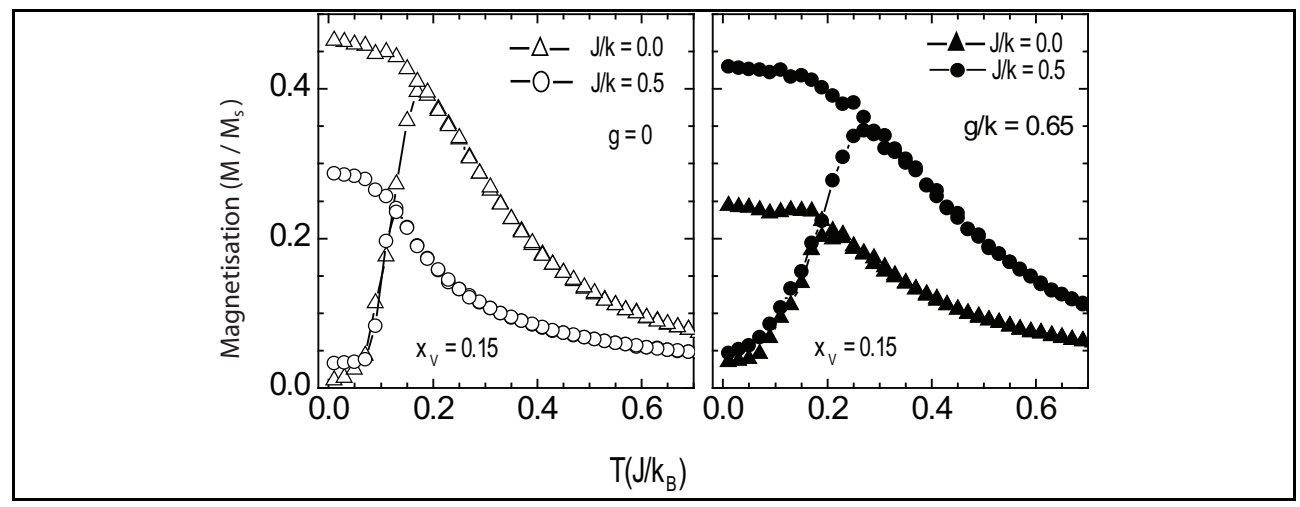

Fig. 12. Temperature dependence of magnetisation (ZFC/FC curves). (a) exchange coupling only $(\mathrm{g}=0)$. (b)exchange and dipolar coupling $(\mathrm{g} / \mathrm{k}=0.65)$. Applied field $\mathrm{h} / \mathrm{k}=0.1$.

\subsection{Ordered arrays of magnetic nanoparticles}

Ordered arrays of magnetic nanoparticles (Petit et al., 1998; Murray et al.,2001; Puntes et al., 2001) and patterned magnetic media (White, 2002) are currently the most promising materials for exploitation in high-density $\left(\sim 1 \mathrm{~Tb} / \mathrm{in}^{2}\right)$ magnetic storage media, due to the sharp distribution of their magnetic properties and their high reproducibility. Nanoparticle arrays (or super lattices) are prepared by colloidal synthesis followed by size-selective precipitation that produces a very narrow particle size distribution $(\sigma<5 \%)$. In addition to their technological applications, nanoparticles superlattices consist the ideal system for studying magnetisation reversal mechanism in the presence of interparticle interactions, due to the precise knowledge and control of the particle size and interparticle distances. Recent studies of self-assembled arrays of magnetic nanoparticles have provided clear evidence that interactions between the nanoparticles are present and manifest themselves in various aspects of their magnetic behaviour.

In figure 13 we show the zero-field cooled (ZFC) magnetisation curves for various coverage (c) values and parameters corresponding to hard Co nanoparticles (Kechrakos \& Trohidou, 2002). The maximum of the $\mathrm{ZFC}$ curve appears at the blocking temperature $\left(\mathrm{T}_{\mathrm{b}}\right)$ of the system (Dormann et al., 1997). An obvious increase of the blocking temperature with layer coverage is seen, that almost reaches saturation as soon as the first complete ML is formed $(\mathrm{c}=1)$. The increase of $\mathrm{T}_{\mathrm{b}}$ with coverage, below $1 \mathrm{ML}$, is due to the anisotropic and ferromagnetic character of dipolar interactions that introduce an additional barrier to the magnetisation reversal of the particles. The increase of $T_{b}$ with particle concentration has 
also been observed in granular films (Dormann et al., 1997; Kechrakos \& Trohidou, 1998; Chrantrell et al., 2001). Increased $\mathrm{T}_{b}$ values relative to the dilute limit have been recently measured in self-assembled Co nanoparticle arrays prepared from colloidal dispersions (Murray et al., 2001) and in self-organized lattices of Co clusters in $\mathrm{Al}_{2} \mathrm{O}_{3}$ matrix (Luis et al., 2002). However, in the latter experiments the saturation of $T_{b}$ values was found after 5-7 layers, while in closed-packed hexagonal arrays we demonstrate that saturation occurs already after two layers. Thus, we conclude that in hexagonal closed packed spherical Co particles the collective behaviour is predominantly determined by the intra-layer dipolar interactions, while inter-layer interactions play only a secondary role.

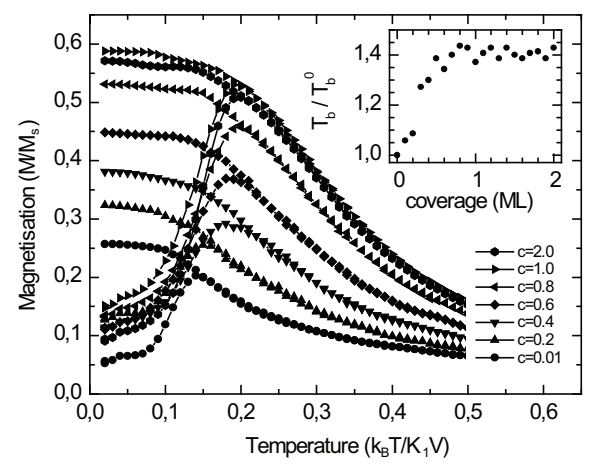

Fig. 13. Dependence of ZFC/FC magnetisation on layer coverage (c) for hard Co nanoparticles $(g / k=0.25)$. Top layer is randomly occupied. In-plane applied field $h / k=0.1$. Inset shows the dependence of blocking temperature on monolayer coverage.

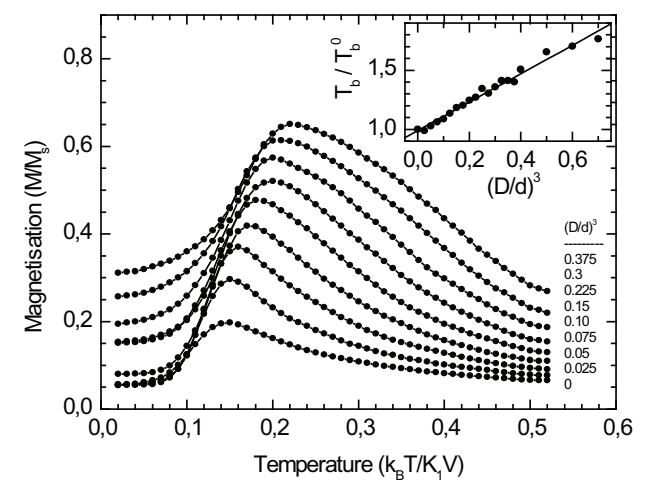

Fig. 14. Dependence of ZFC magnetisation on interparticle distance for hard Co nanoparticles $(\mathrm{g} / \mathrm{k}=0.25)$. Uppermost layer is randomly occupied. In-plane applied field $\mathrm{h} / \mathrm{k}=0.1$. Inset shows the scaling behaviour of blocking temperature with interparticle distance. 
A strong dependence of the $\mathrm{T}_{\mathrm{b}}$ on the interparticle distance $(\mathrm{d})$ is shown in figure 14. Our data indicate that for hard magnetic nanoparticles $(\mathrm{g} / \mathrm{k}<1)$ the blocking temperature scales with the inverse cube of the interparticle distance, $\mathrm{T}_{\mathrm{b}} \sim(\mathrm{D} / \mathrm{d})^{3}$ (Kechrakos \& Trohidou, 2002). Bearing in mind that the dipolar strength is $g \sim(D / d)^{3}$, we obtain $T_{b} \sim g$, which means that in hexagonal arrays of magnetically hard nanoparticles the dipolar interactions provide an additional energy barrier for magnetisation reversal which is proportional to the dipolar coupling strength. Our results are in qualitative agreement with recent measurements of ZFC/FC curves (Murray et al., 2001; Luis et al., 2002) indicating an increase of $T_{b}$ relative to the dilute limit.

\section{Concluding remarks}

We have reviewed Monte Carlo simulation results on the magnetic behavior of nanoparticles and nanoparticle assemblies. Our results show that the MC simulation technique with the implementation of the Metropolis algorithm is an indispensable tool for the study of these systems, because they can include implicitly the details of the interface and the surface for the single nanoparticles and in all studied cases the temperature.

Our simulations have shown the dominant role of the surface to the magnetic behavior of antiferromagnetic and ferrimagnetic nanoparticles due to its anisotropy as well as the number of 'uncompensated' magnetic moments. The behavior of their coercive field $\mathrm{H}_{\mathrm{c}}$ depends also on the details of their surface and on their size. The comparison between our simulation results and the experimental findings for antiferromagnetic nanoparticles $\mathrm{NiO}$ or the spherical and cubic ferrimagnetic nanoparticles $\gamma-\mathrm{Fe}_{2} \mathrm{O}_{3}$ confirmed the important role of the surface and the uncompensated spins to the magnetic behavior of nanoparticles.

Our study was also extended to the case of composite nanoparticles with ferromagnetic core and a ferrimagnetic or antiferromagnetic shell. We demonstrated the important role of the interface in their magnetic behavior. The training and aging effects, which are characteristic of disordered systems, have been studied for the composite nanoparticles. The simulation results are in agreement with the experimental findings.

We demonstrated via Monte Carlo simulations in the case of the three-dimensional random assembly of interacting nanoparticles coupled via exchange and dipolar forces that the coercivity decreases with concentration for all values of the exchange strength and the blocking temperature increases with concentration, except when particle coalescence occurs and the system is above the percolation limit.

In the case of ordered arrays of nanoparticles our simulations showed that the blocking temperature increases with the coverage of the first monolayer and is rather insensitive to additional monolayers and decreases proportionally to the cube of the interparticle distance $\left(\mathrm{T}_{\mathrm{b}} \sim 1 / \mathrm{d}^{3}\right)$.

There are still several interesting issues to be addressed in the field of magnetic nanoparticles. The magnetic behaviour of complex inverted core/shell nanostructures. The influence of interparticle interactions on the magnetic properties of nanoparticles in dense nanoparticle assemblies is of major importance especially for the technological exploitation of magnetic nanoparticle assemblies. The interparticle interaction effects in nanoparticle assemblies with core/shell morphology are a new open field for study. MC simulations provide an extremely valuable tool for the study of these systems. 


\section{Acknowledgements}

We would like to acknowledge the contribution of Dr. J.A. Blackman to parts of the work presented in this chapter and the helpful discussions with Dr. D. Fiorani, Prof. J. Nogués and Prof. C. Binns. This work was supported by the project of the Greek General Secreteriat of Research PENED96, and the EC projects AMMARE (Contract No G5RD-CT-2001-00478) and NANOSPIN (Contract No NMP4-CT-2004-013545).

\section{References}

Bader, S. D. (2006). Colloquium: Opportunities in nanomagnetism. Reviews of Modern Physics, Vol.78, No.1, (January 2006), pp. 1-15, ISSN 0034-6861

Bahiana,M.; Pereira Nunes, J.P.; Altbir,D.; Vargas, P. \& Knobel, M. (2004). Ordering effects of the dipolar interaction in lattices of small magnetic particles. Journal of Magnetism and Magnetic Materials, Vol.281, No.2-3, (October 2004), pp. 372-377, ISSN 0304-8853

Baker, C.; Hasanain, S. K. \& Shah, S. I. (2004). The magnetic behavior of iron oxide passivated iron nanoparticles. Journal of Applied Physics, Vol.96, No.11, (December 2004), pp. 6657-6666, ISSN 0021-8979

Bedanta, S.; Eimuller, T.; Kleemann, W.; Rhensius, J.; Stromberg, F. ; Amaladass, E.; Cardoso, S. ; Freitas, P.P. (2007). Overcoming the dipolar disorder in dense CoFe nanoparticle ensembles: Superferromagnetism. Physical Review Letters, Vol.98, No. 17, (April 2007), pp. 176601, ISSN 1079-7114

Bhowmik, R. N.; Nagarajan, R. \& Ranganathan, R. (2004). Magnetic enhancement in antiferromagnetic nanoparticle of CoRh2O4. Physical Review B, Vol.69, No.5, (February 2004), pp. 054430-5, ISSN 1098-0121

Binder, K.; Rauch, H. \& Wildpaner, H. (1970). Monte Carlo calculation of the magnetisation of superparamagnetic particles. Journal of Physics and Chemistry of Solids, Vol.31, No.2, (February 1970), pp. 391-397, ISSN 0022-3697

Binder, K. (1986). Topics in Current Physics, Monte Carlo Methods in Statistical Physics, Springer-Verlag, ISBN: 3-540-16514-2, Berlin Heidelberg New York Tokyo

Binder, K. \& Young, A. P. (1986). Spin glasses: Experimental facts, theoretical concepts, and open questions. Reviews of Modern Physics, Vol.58, No.4, (October-December 1986), pp. 801-976, ISSN 0034-6861

Binder, K. (1987). Applications of the Monte-Carlo Method in Statistical Physics, Springer-Verlag, ISBN: 3540176500, New York

Binns, C.; Maher, M.J.; Pankhurst, Q. A. ; Kechrakos, D. \& Trohidou, K.N. (2002). Magnetic behavior of nanostructured films assembled from preformed Fe clusters embedded in Ag. Physical Review B, Vol.66, No.18, (November 2002), pp. 184413-12, ISSN 10980121

Bødker, F. ; Mørup, S. \& Linderoth, S. (1994). Surface effects in metallic iron nanoparticles. (1994) Surface effects in metallic iron nanoparticles. Physical Review Letters, Vol.72, No.2, (January1994), pp. 282-285, ISSN 1079-7114

Brown, G.; Janotti, A.; Eisanbach, M. \& Stocks, G. M. (2005). Intrinsic volume scaling of thermoinduced magnetisation in antiferromagnetic nanoparticles. Physical Review B, Vol.72, No.14, (October 2005), pp. 140405R-4, ISSN 1098-0121 
Chamberlin, R.V.; Mozurkewich, V.; Orbach, R. (1984). Time Decay of the Remanent Magnetisation in Spin-Glasses. Physical Review Letters, Vol.52 No.10, (March 1984), pp. 867-870, ISSN 1079-7114

Chen, C.; Kitakami, O. \& Shimada, Y. (1998). Particle size effects and surface anisotropy in Fe-based granular films. Journal of Applied Physics, Vol.84, No.4, (August 1998), pp. 2184-2188, ISSN 0021-8979

Chantrell, R. W.; Walmsley, N., Gore, J. \& Maylin, M. (2001). Calculations of the susceptibility of interacting superparamagnetic particles. Physical Review B, Vol.63, No.2, (January 2001), pp. 024410-14, ISSN 1098-0121

Coey, J. M. D. (1971). Noncollinear Spin Arrangement in Ultrafine Ferrimagnetic Crystallites. Physical Review Lettetes, Vol.27, No.17, (October 1971), pp. 1140-1142, ISSN 1079-7114

Curiale, J. ; Granada, M. ; Troiani, H. E. ; Sánchez, R. D. ; Leyva, A. G. ; Levy, P. \& Samwer, K. (2009). Magnetic dead layer in ferromagnetic manganite nanoparticles. Applied Physics Letters, Vol.95, No.4, (July 2009), pp. 043106-3, ISSN 0003-6951

De Biasi, E.; Ramos, C. A. ; Zysler, R. D. \& Romero, H. (2002). Large surface magnetic contribution in amorphous ferromagnetic nanoparticles. Physical Review B, Vol.65, No.14, (March 2002), pp. 144416-8, ISSN 1098-0121

Del Bianco, L.; Fiorani, D. ; Testa, A. M.; Bonetti, E. ; Savini, L. \& Signoretti, S. (2003). Magnetic properties of the $\mathrm{Fe} / \mathrm{Fe}$ oxide granular system. Journal of Magnetism and Magnetic Materials, Vol.262, No.1, (May 2003), pp. 128-131, ISSN 0304-8853

Del Bianco, L. D.; Fioarani, D.; Testa, A. M.; Bonetti, E. \& Signorini, L. (2004). Field-cooling dependence of exchange bias in a granular system of Fe nanoparticles embedded in an Fe oxide matrix. Physical Review B, (August 2004), pp. 052401 -4, ISSN 1098-0121

Dimitrov, D. A. \& Wysin, G. M. (1994). Effects of surface anisotropy on hysteresis in fine magnetic particles. Physical Review B, Vol.50, No.5, (August 1994), pp. 3077-3084, ISSN 1098-0121

Dimitrov, D. A. \& Wysin, G. M. (1996). Magnetic properties of superparamagnetic particles by a Monte Carlo method. Physical Review B, Vol.54, No.13, (October 1996), pp. 9237-9241, ISSN 1098-0121

Dormann, J. L., Fiorani, D. and Tronc, E. (1997). Magnetic Relaxation in Fine-Particle Systems, in Advances in Chemical Physics, Vol.98, pp. 283-494 (eds I. Prigogine and S. A. Rice), John Wiley \& Sons, Inc., Hoboken, New York, USA Book Series: Advances in Chemical Physics, ISBN: 9780471162858

Du, H. F. \& Du, A. (2006). The hysteresis curves of nanoparticles obtained by Monte Carlo method based on the Stoner-Wohlfarth model. Journal of Applied Physics, Vol.99, No.10, (May 2006), pp. 104306-4, ISSN 0021-8979

Eftaxias, E. \& Trohidou, K. N. (2005). Numerical study of the exchange bias effects in magnetic nanoparticles with core / shell morphology. Physical Review B, Vol.71, No.13, (April 2005), pp. 134406-6, ISSN 1098-0121

Eftaxias, E.; Vasilakaki, M. \& Trohidou, K. N. (2007). A Monte Carlo study of the exchange bias effects in magnetic nanoparticles with ferromagnetic core/ antiferromagnetic shell morphology. Modern Physics Letters B, Vol.21, No. 21, (August 2007), pp. 11691177, ISSN: 0217-9849

Fiorani, D., Del Bianco, L.; Testa, A.M. \& Trohidou, K.N. (2006). Glassy dynamics in the exchange bias properties of the iron/iron oxide nanogranular system. Physical Review B, Vol.73, No.9, (March 2006), pp. 092403, ISSN 1098-0121 
Gangopadhyay, S.; Hadjipanayis, G. C. ; Dale, B. ; Sorensen, C. M. ; Klabunde, K. J. ; Papaefthymiou, V. \& Kostikas, A. (1992). Magnetic properties of ultrafine iron particles. Physical Review B, Vol.45, No.17, (May 1992), pp. 9778-9787, ISSN 10980121

Garanin, D. A. \& Kachkachi, H. (2003). Surface Contribution to the Anisotropy of Magnetic Nanoparticles. Physical Review Letters, Vol.90, No.6, (February 2003), pp. 065504-4, ISSN 1079-7114

Garcia-Otero, J.; Porto, M.; Rivas, J. \& Bunde, A. (1999). Influence of the cubic anisotropy constants on the hysteresis loops of single-domain particles: A Monte Carlo study. Journal of Applied Physics, Vol.85, No.4, (February 1999), pp. 2287-2292, ISSN 00218979

Grzybowski, A.; Gwóźdź, E. \& Bródka, A. (2000). Ewald summation of electrostatic interactions in molecular dynamics of a three-dimensional system with periodicity in two directions. Physical Review B, Vol.61, No.10, (March 2000), pp. 6706-6712, ISSN 1098-0121

Hinzke, D. \& Nowak., U. (1999). Monte Carlo simulation of magnetisation switching in a Heisenberg model for small ferromagnetic particles. Computer Physics Communications, Vol.121-122, (September-October 1999), pp. 334-337, ISSN 00104655

Iglesias, O. \& Labarta, A. (2004). Shape and surface anisotropy effects on the hysteresis of ferrimagnetic nanoparticles. Journal of Magnetism and Magnetic Materials, Vol.272276, No.1, (May 2004), pp. 685-686, ISSN 0304-8853

Jamet, M.; Wernsdorfer, W.; Thirion, C.; Mailly, D. ; Dupuis, V. ; Mélinon, P. \& Pérez, A. (2001). Magnetic Anisotropy of a Single Cobalt Nanocluster. Physical Review Letters, Vol.86, No.20, (June 2001), pp. 4676-4679, ISSN 1079-7114

Kachkachi, H.; Nogues, M.; Tronc, E. \& Garanin, D. A. (2000). Finite-size versus surface effects in nanoparticles. Journal of Magnetism and Magnetic Materials, Vol.221, No.12, (November 2000), pp. 158-163, ISSN 0304-8853

Kechrakos, D. \& Trohidou, K. N. (1998). Magnetic properties of dipolar interacting singledomain particles. Physical Review B, Vol.58, No.18, (November 1998), pp. 1216912177, ISSN 1098-0121

Kechrakos, D. \& Trohidou, K. N. (2002). Magnetic properties of self-assembled interacting nanoparticles. Applied Physics Letters, Vol.81, No.24, (December 2002), pp. 45744576, ISSN 0003-6951

Kechrakos, D. \& Trohidou, K. N. (2003). Competition between dipolar and exchange interparticle interactions in magnetic nanoparticle films. Journal of Magnetism and Magnetic Materials, Vol.262, No.1, (May 2003), pp. 107-110, ISSN 0304-8853

Kneller, E. F. \& Luborsky, F. E. (1963). Particle Size Dependence of Coercivity and Remanence of Single-Domain Particles. Journal of Applied Physics, Vol.34, No.3, (March 1963), pp. 656-659, ISSN 0021-8979

Kodama, R. H. (1999). Magnetic nanoparticles. Journal of Magnetism and Magnetic Materials, Vol.200, No.1-3, (October 1999), pp. 359-372, ISSN 0304-8853

Kretschmer, R. \& Binder, K. (1979). Ordering and phase transitions in Ising systems with competing short range and dipolar interactions. Zeitchrift für Physik B, Vol.34, No.4, (December 1979), pp. 375-392, ISSN 0722-3277 
Landau, D. P. (1976). Finite-size behavior of the Ising square lattice. Physical Review B, Vol.13, No.7, (April 1976), pp. 2997-3011, ISSN 1098-0121

Landau, D. P. \& Binder, K. (2000). A Guide to Monte Carlo Simulations in Statistical Physics, Gambridge University Press, ISBN: 052165314 2, U. K.

Leighton, C.; Nogues, J. ; Jonsson-Akerman, B. J. \& Schuller, I. K. (2000). Coercivity Enhancement in Exchange Biased Systems Driven by Interfacial Magnetic Frustration. Physical Review Letters, Vol.84, No.15, (April 2000), pp. 3466-3469, ISSN 1079-7114

Leite, V. S.; Godoy, M. \& Figueiredo, W. (2005). Finite-size effects and compensation temperature of a ferrimagnetic small particle. Physical Review B, Vol.71, No.9, (March 2005), pp. 094427 -7, ISSN 1098-0121

Lin, X.; Murthy, A. S.; Hadjipanayis, G. C. ; Swann, C. \& Shah, S. I. (1994). Magnetic and structural properties of Fe-FeO bilayers. Journal of Applied Physics, Vol.76, No.10, (November 1994 ), pp. 6543 -6546, ISSN 0021-8979

Luis, F.; Petroff, F.; Torres, J.M.; Garcia, L.M.; Bartolome, J.; Carrey, J. \& Vaures, A.(2002). Magnetic relaxation of interacting Co clusters: Crossover from two- to threedimensional lattices. Physical Review Letters, Vol.88, No.21, (May 2002), pp. 217205, ISSN 0031-9007

Martinez, B.; Obradors, X. ; Balcells, Ll.; Rouanet, A. \& Monty, C. (1998). Low Temperature

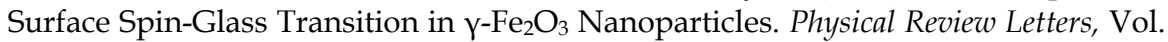
80, No.1, (January 1998), pp. 181-184, ISSN 1079-7114

Meiklejohn, W. H. \& Bean, C. P. (1957). New Magnetic Anisotropy. Physical Review, Vol. 105, No.3, (February 1957), pp. 904-913, ISSN 0031-899X

Metropolis, N.; Rosenbluth, A.; Rosenbluth, M.; Teller, A. \& Teller, E. (1953). Equation of State Calculations by Fast Computing Machines. Journal of Chemical Physics, Vol.21, No.6, (June 1953), pp. 1087-1092, ISSN 0021-9606

Morel, R.; Brenac, A. \& Portemont, C. (2004). Exchange bias and coercivity in oxygenexposed cobalt clusters. Journal of Applied Physics, Vol.95, No.7, (April 2004), pp. 3757-3761, ISSN 0021-8979

Mørup, S. \& Hansen, B. R. (2005). Uniform magnetic excitations in nanoparticles. Physical Review B, Vol.72, No.2, (July 2005), pp. 024418-6, ISSN 1098-0121

Mørup, S.; Madsen, D. E.; Frandsen, C.; Bahl C. R. H. \& Hansen, M. F. (2007). Experimental and theoretical studies of nanoparticles of antiferromagnetic materials. Journal of Physics: Condensed Matter, Vol.19, No.21, (May 2007), pp. 213202 -212333, ISSN 09538984

Murray, C. B.; Sun, S.H.; Doyle, H.; Betley, T. (2001). Monodisperse 3d transition-metal (Co, $\mathrm{Ni}, \mathrm{Fe}$ ) nanoparticles and their assembly into nanoparticle superlattices. Materials Research Society Bulletin, Vol.26, No.12, (December 2001), pp. 985-991

Néel, L. (1953). Some New Results on Antiferromagnetism and Ferromagnetism. Reviews of Modern Physics, Vol.25, No.1, (January-March 1953), pp. 58-63, ISSN 1539-0756

Nogués, J. \& Schuller, I. K. (1999). Exchange bias. Journal of Magnetism and Magnetic Materials, Vol.192, No.2, (February 1999), pp. 203-232, ISSN 0304-8853

Nogués, J.; Sort, J.; Langlais, V.; Skymryev, V.; Surinach, S,; Munoz, J. \& Baro, M. D. (2005). Exchange bias in nanostructures. Physics Reports, Vol.422, No.3, (December 2005), pp. 65-117, ISSN: 0370-1573 
Nogués, J.; Skumryev, V.; Sort, J. ; Stoyanov, S. \& Givord, D. (2006). Shell-Driven Magnetic Stability in Core-Shell Nanoparticles. Physical Review Letters, Vol.97, No.15, (October 2006), pp. 157203-4, ISSN 1079-7114

Pankhurst, Q. A.; Connolly, J.; Jones, S. K. \& Dobson, J. (2003). Applications of magnetic nanoparticles in biomedicine. Journal of Physics D: Applied Physics, Vol.36, No.13, (June 2003), pp. R167-181, ISSN 0022-3727

Passamani, E. C.; Larica, C.; Marques, C.; Proveti, J. R.; Takeuchi, A. Y. \& Sanchez, F. H. (2006). Exchange bias and anomalous vertical shift of the hysteresis loops in milled $\mathrm{Fe} / \mathrm{MnO} 2$ material. Journal of Magnetism and Magnetic Materials, Vol.299, No.1, (April 2006), pp. 11-20, ISSN 0304-8853

Peng, D.L.; Hihara, T.; Sumiyama, K.; Morikawa, H. (2002). Structural and magnetic characteristics of monodispersed Fe and oxide-coated Fe cluster assemblies. Journal of Applied Physics, Vol.92, No.6, (September 2002), pp. 3075-3083, ISSN 0021-8979

Petit, C. ; Taleb, A.; Pileni, M.P. (1998). Self-organization of magnetic nanosized cobalt particles. Advanced Materials, Vol.10, No.3, (February 1998), pp. 259-261, ISSN 15214095

Puntes,V. F.; Krishnam, K. M. \&Alivisatos, A. P. (2001). Synthesis, self-assembly, and magnetic behavior of a two-dimensional superlattice of single-crystal $\varepsilon$-Co nanoparticles. Applied Physics Letters, Vol.78, No.15, (April 2001), pp. 2187-2190, ISSN 0003-6951

Respaud, M.; Broto, J. M.; Rakoto, H.; Fert, A. R.; Thomas, L. ; Barbara, B.; Verelst, M.; Snoeck, E.; Lecante, P. ; Mosset, A. ; Osuna, J. ; Ould Ely, T.; Amiens, C. \& Chaudret, B. (1998). Surface effects on the magnetic properties of ultrafine cobalt particles. Physical Review B, Vol.57, No.5, (February 1998), pp. 2925-2935, ISSN 10980121

Sahoo, S.; Petracic, O.; Kleemann, W.; Stappert, S. ; Dumpich, G. ; Nordblad, P.; Cardoso, S.; Freitas, P.P. (2003). Cooperative versus superparamagnetic behavior of dense magnetic nanoparticles in $\mathrm{Co}_{80} \mathrm{Fe}_{20} / \mathrm{Al}_{2} \mathrm{O}_{3}$ multilayers. Applied Physics Letters, Vol. 82, No.23, (June 2003), pp. 4116-4118, ISSN 0003-6951

Sinohora, T.; Sato, T.; Taniyama, T. \& Nakatani. I. (1999). Size dependent magnetisation of PdFe fine particles . Journal of Magnetism and Magnetic Materials, Vol.196-197, (May 1999), pp. 94-95, ISSN 0304-8853

Skumryev, V.; Stoyanov, S.; Zhang, Y.; Hadjipanayis, G.; Givord, D. \& Nogués J. (2003). Beating the superparamagnetic limit with exchange-bias. Nature (London), Vol.423, No.6942, (June 2003), pp. 850-853, ISSN 0028-0836

Stoner, E. C. \& Wohlfarth, E. P. (1948). A Mechanism of Magnetic Hysteresis in Heterogeneous Alloys. Philosophical Transactions of the Royal Society London, Series A, Vol.240, No.826, (May 1948), pp. 599 - 642, Online ISSN: 1471-2962

Trohidou, K. N.; Zianni, X. \& Blackmann, J. A. (1998). Surface effects on the magnetic behavior of antiferromagnetic particles. Journal of Applied Physics, Vol.84, No.5, (September 1998), pp. 2795-2801, ISSN 0021-8979

Trohidou, K. N. (2005). Monte Carlo studies of surface and interface effects in magnetic nanoparticles, In: Surface Effects in Magnetic Nanoparticles (Nanostructured science and technology), Fiorani, D., pp. 45-74, Springer, ISBN 0-387-23279-6, N. York, U. S. A.

Trohidou, K.N.; Vasilakaki, M.; Del Bianco, L.; Fiorani, D. \& Testa, A.M. (2007). Exchange bias in a magnetic ordered/disordered nanoparticle system A Monte Carlo 
simulation study. Journal of Magnetism and Magnetic Materials, Vol.316, No.2, (September 2007), pp. e82-e87, ISSN 0304-8853

Vasilakaki, M. \& Trohidou, K. N. (2008). Surface effects on the magnetic behavior of nanoparticles with core / shell morphology. Journal of Physics D: Applied Physics, Vol.41, No.13, (July 2008), pp. 134006-5, ISSN 0022-3727

Vasilakaki, M. \& Trohidou, K. N. (2009). Numerical study of the exchange-bias effect in nanoparticles with ferromagnetic core / ferrimagnetic disordered shell morphology. Physical Review B, Vol.79, No.14, (April 2009), pp. 144402-8, ISSN 10980121

Wildpaner, V. (1974). Monte Carlo study of small magnetic particles. Zeitchrift für Physik A, Vol.270, No.3, (February 1974), pp. 215-223, ISSN 0939-7922

Winkler, E.; Zysler, R. D.; Vasquez Mansilla, M,; Fiorani, D.; Rinaldi, D.; Vasilakaki, M. \& Trohidou, K. N. (2008). Surface spin-glass freezing in interacting core-shell NiO nanoparticles. Nanotechnology, Vol.19, No.18, (May 2008), pp. 185702-185710, ISSN 0957-4484

White, R.L. (2002). Magnetisation processes in patterned media. Journal of Magnetism and Magnetic Materials, Vol.242, No.1, (April 2002), pp. 21-26, ISSN 0304-8853

Zianni, X. \& Trohidou, K. N. (1998). Monte Carlo simulations on the coercive behaviour of oxide coated ferromagnetic particles. Journal of Physics: Condensed Matter, Vol.10, No.33, (August 1998), pp. 7475-7483, ISSN 0953-8984 


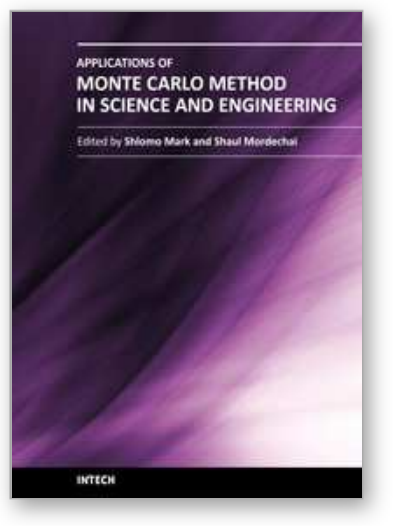

\section{Applications of Monte Carlo Method in Science and Engineering \\ Edited by Prof. Shaul Mordechai}

ISBN 978-953-307-691-1

Hard cover, 950 pages

Publisher InTech

Published online 28, February, 2011

Published in print edition February, 2011

In this book, Applications of Monte Carlo Method in Science and Engineering, we further expose the broad range of applications of Monte Carlo simulation in the fields of Quantum Physics, Statistical Physics, Reliability, Medical Physics, Polycrystalline Materials, Ising Model, Chemistry, Agriculture, Food Processing, X-ray Imaging, Electron Dynamics in Doped Semiconductors, Metallurgy, Remote Sensing and much more diverse topics. The book chapters included in this volume clearly reflect the current scientific importance of Monte Carlo techniques in various fields of research.

\section{How to reference}

In order to correctly reference this scholarly work, feel free to copy and paste the following:

K. Trohidou and M. Vasilakaki (2011). Monte Carlo Studies of Magnetic Nanoparticles, Applications of Monte Carlo Method in Science and Engineering, Prof. Shaul Mordechai (Ed.), ISBN: 978-953-307-691-1, InTech, Available from: http://www.intechopen.com/books/applications-of-monte-carlo-method-in-science-andengineering/monte-carlo-studies-of-magnetic-nanoparticles

\section{INTECH}

open science | open minds

\section{InTech Europe}

University Campus STeP Ri

Slavka Krautzeka 83/A

51000 Rijeka, Croatia

Phone: +385 (51) 770447

Fax: +385 (51) 686166

www.intechopen.com

\section{InTech China}

Unit 405, Office Block, Hotel Equatorial Shanghai

No.65, Yan An Road (West), Shanghai, 200040, China

中国上海市延安西路65号上海国际贵都大饭店办公楼 405 单元

Phone: +86-21-62489820

Fax: $+86-21-62489821$ 
(C) 2011 The Author(s). Licensee IntechOpen. This chapter is distributed under the terms of the Creative Commons Attribution-NonCommercialShareAlike-3.0 License, which permits use, distribution and reproduction for non-commercial purposes, provided the original is properly cited and derivative works building on this content are distributed under the same license. 